\title{
ECOTONE SOILS IN NORTHEASTERN BRAZIL
}

José João Lelis Leal de Souza

Universidade Federal de Viçosa - UFV

Departamento de Solos, Viçosa, MG, Brasil

jilelis@ufv.br

Anderson Silva Pinto

Universidade Estadual da Paraíba - UEPB

Laboratório de Ecologia Neotropical, Campina Grande, PB, Brasil anderson.slvp@gmail.com

Maiara Bezerra Ramos

Universidade Estadual da Paraíba - UEPB

Laboratório de Ecologia Neotropical, Campina Grande, PB, Brasil maiarabramos@gmail.com

Sérgio de Faria Lopes

Universidade Estadual da Paraíba - UEPB

Laboratório de Ecologia Neotropical, Campina Grande, PB, Brasil defariaslopes@gmail.com

\begin{abstract}
Texture, base saturation, organic carbon content, and water storage availability of soil are drivers of plant physiognomy and composition of communities. Soil properties in ecotone areas are still poorly studied, and the transition between dry, moist, and semideciduous forests is defined only by climate parameters. The objective of this study was to describe the soil properties of a moist-dry forest ecotone in Northeastern Brazil. Seven soil profiles were dug in a pristine semideciduous forest known as "Agreste". Four more pedons were described to represent soils of dry forests. Morphology, reactivity, texture, organic matter content, and water storage capacity of the soil horizons were determined. The soils of the study area are derived from granites and granitoids, rocks highly resistant to weathering. Soils of dry forests are loam, neutral to alkaline, and hypereutrophic. Soils of semideciduous forest are sandy, acidic, dystrophic, and have up to $65 \%$ higher $\mathrm{C}$ content. The rocks act as impermeable layers to water, and consequently, most soils develop stagnic properties in semideciduous forests. Soils are dystrophy and have low CEC and loam texture. These properties are attributed to ferrolysis. Umbrisols and Stagnosols with higher water storage capacity than dry forests soils sustain semideciduous forests in Northeastern Brazil.
\end{abstract}

Keywords: Caatinga. Semideciduous forest. Dry forest. Water storage.

\section{SOLOS ECOTONAIS NO NORDESTE DO BRASIL}

\section{RESUMO}

Textura, saturação de base, conteúdo de carbono orgânico e disponibilidade de armazenamento de água do solo são fatores determinantes da fisionomia das plantas e composição das comunidades. As propriedades do solo em áreas de ecótonos ainda são pouco estudadas e a transição entre florestas secas, úmidas e semidecíduas é definida apenas por parâmetros climáticos. O objetivo deste estudo foi descrever as propriedades do solo de um ecótono de floresta úmida e seca no Nordeste do Brasil. Sete perfis de solo foram descritos em uma floresta semidecídua intocada conhecida como "Agreste". Mais quatro perfis foram descritos para representar solos de florestas secas. A morfologia, reatividade, textura, conteúdo de matéria orgânica e capacidade de armazenamento de água dos horizontes do solo foram determinadas. Os solos das florestas secas são de textura média, neutros a alcalinos e eutróficos. Os solos da floresta semidecidual são arenosos, ácidos, distróficos e têm até $65 \%$ maior teor de C. As rochas atuam como camadas impermeáveis à água e, consequentemente, a maioria dos solos desenvolve 
cores gleizadas em florestas semideciduais. Os solos são distróficos e possuem baixa CTC e textura média. Essas propriedades são atribuídas à ferrólise. Neossolos e Gleissolos com maior capacidade de armazenamento de água em comparação com solos de florestas secas sustentam florestas semideciduais no Nordeste do Brasil.

Palavras-chave: Caatinga. Floresta estacional semidecidual. Floresta seca. Armazenamento de água.

\section{INTRODUCTION}

The classification of vegetation types, or biomes, is an issue that is currently debated (ARRUDA et al., 2017; PENNINGTON; LEHMANN; ROWLAND, 2018; MUCINA, 2019). In tropical zones, the occurrence of moist and dry forests is associated with the climate (PENNINGTON; LEHMANN; ROWLAND, 2018), edaphic factor, and vegetable composition (MIRANDA et al., 2018). The transition between moist and dry forest in Northeastern Brazil is called "Agreste'. This zone is a zonal forest that comprises six states in Brazil and covers more than 100,343 km² (IBGE, 2010). Semideciduous forests cover this land and are mostly used for agriculture, prevailing fruits. Like the Caatinga, the Agreste is frequently affected by drought, though generally with less severe effects.

The definition of semideciduous forests is still uncertain. Some studies have grouped semideciduous forests with the dry forest biome (PENNINGTON et al., 2000) and others with the moist forest biome (BANDA et al., 2016). Nowadays, semideciduous forests are described as transition zones between dry and moist forests in the Neotropics (DEXTER et al., 2018). Vegetable composition and abiotic factors, such as climate and soil properties, define their occurrence (PENNINGTON et al., 2018). Although semideciduous forests are expected to cover approximately 1 million $\mathrm{km}^{2}$ in South America (DEXTER et al., 2018), its occurrence is still poorly defined (PENNINGTON et al., 2000; MIRANDA et al., 2018).

The average annual precipitation, potential evapotranspiration, and seasonality are important factors responsible for delimiting moist forests, dry forests, and savannas (PENNINGTON et al., 2018). Besides, water availability and fertility of the soils also play critical roles (ARRUDA et al., 2015; de MENDONÇA et al., 2017; RODRIGUES et al., 2018) in the selection of vegetation composition. The soil influences plant physiognomies in the Brazilian Savanna (Cerrado) (NERI et al., 2013), Restinga forest (MAGNAGO et al., 2012), Amazonia rainforest (SOUZA et al., 2018; HOORN et al., 2010), Tundra (POELKING et al., 2015) and other biomes around the world (ALLIÉ et al., 2015; JONES et al., 2016). Although soil properties are essential for vegetation, they have received limited attention in studies regarding biome transitions.

The occurrence of semideciduous forests is still blurry once its delimitation depends on climate conditions, relief, and soil properties (PENNINGTON et al., 2018). Thus, we have hypothesized that specific soil properties are related to the occurrence of semideciduous forests. This study aimed to characterize the soil properties in a gradient slope at a preserved hill in semideciduous forests in Northeastern Brazil.

\section{METHODOLOGY}

\section{Study area}

The study area encompasses the Borborema province in the State of Paraíba (Figure 1). The Borborema province has a total area of approximately $25.984 \mathrm{~km}^{2}$ and specific landscape diversity. Altitudes vary between 200 and $1,200 \mathrm{~m}$ in cliffs associated with resistant rocks and horst-graben systems. The Borborema province can be divided into two climatic regions (KAYANO and ANDREOLI, 
2009): i) the Eastern slope, characterized by the occurrence of high orographic rainfalls $(950-1,350$ $\mathrm{mm}$ per year), caused by the range of cliffs with medium altitudes $(600-1,200 \mathrm{~m})$ acting as orogenic barriers to moist fronts of air from the Atlantic Ocean where plant physiognomy is described as a semideciduous forest, which has few endemic tree species and, instead, it contains species of trees associated to dry and moist forests (DEXTER et al., 2018), and; ii) an extensive flat surface, developed on Meso- and Neoproterozoic gneiss and schist, with an average temperature of $23^{\circ} \mathrm{C}$ and precipitation below $700 \mathrm{~mm}$ per year.

Figure 1 - Borborema province: sampling sites according to altitude.

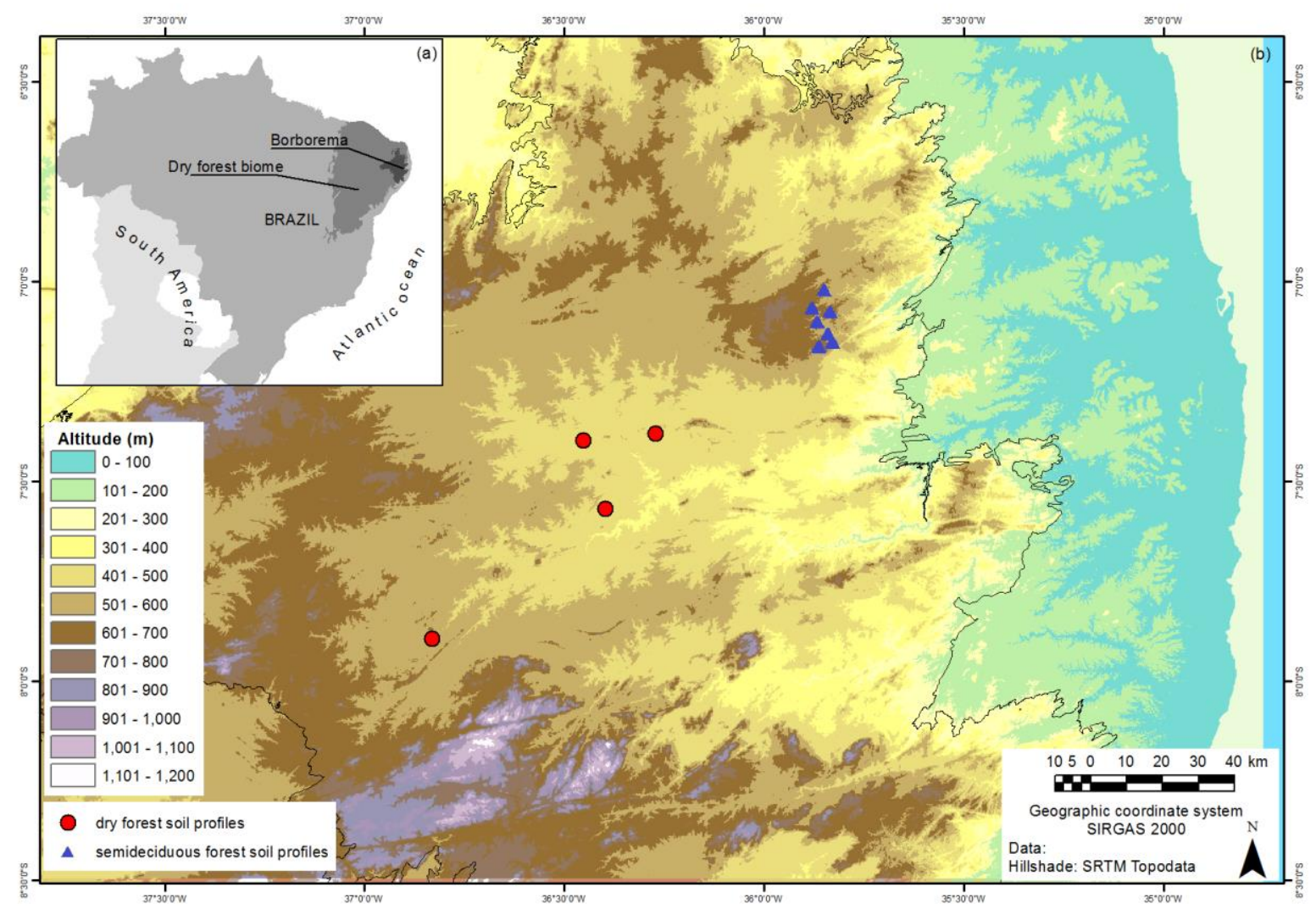

The plant physiognomy is described as a dry forest, composed by Bromeliaceae Juss., Cactaceae Juss. And Fabaceae Lindl. and members of the families Poaceae Barnhart and Cyperaceae Juss. (BANDA et al., 2016). Pollen records and radiocarbon analysis indicate a similar vegetation composition at $\sim 8,500 \mathrm{cal}$ yr B.P. in the extensive flat surface of Borborema province due to semiarid climate establishment and moist forest retraction (de Medeiros et al., 2018). Previous studies indicated the dominance of shallow and incipient weathered soils, with Leptosols (Neossolos Litólicos according to Brazilian Soil Classification System) and Luvisols (Luvissolos Crômicos) representing $43 \%$ of the study area (SANTOS et al., 2011).

\section{Sampling and analysis}

Eleven pedons were taken, described, and classified according to the World Reference Base for Soil Resources (IUSS WORKING GROUP WRB, 2015) and the Brazilian Soil Classification System (SANTOS et al., 2018) to represent soil diversity in the Borborema province. Soil samples were collected from the surface, down to the lithic contact at each pedon. For deeper soils, a 150-cm control

$\begin{array}{llll}\text { Caminhos de Geografia } \quad \text { Uberlândia-MG } & \text { v. 22, n. } 81 \quad \text { jun./2021 } & \text { p. 308-328 Página } 310\end{array}$ 
section was used. Four soil profiles were selected from an open-source database to represent the soil diversity of the Atlantic rainforest (EMBRAPA, 2017).

Samples were air-dried and sieved through a 2-mm sieve before texture, reaction, and organic matter analysis. All procedures were executed according to well-established methods for tropical soils (DONAGEMA et al., 2011). Particle size composition was determined by the sieve-pipette method. The $\mathrm{pH}$ was measured in soil-water (1:2.5) and soil- $\mathrm{KCl}(1: 2.5)$ solutions. Exchangeable $\mathrm{Ca}, \mathrm{Mg}$, and Al were extracted with a $\mathrm{KCl}$ mol L-1 solution. $\mathrm{K}^{+}$and $\mathrm{Na}^{+}$were extracted with Mehlich-1 solution. The potential acidity $(\mathrm{H}+\mathrm{Al})$ was determined by the ammonium acetate method at $\mathrm{pH} 7$. The sum of bases $\left(\mathrm{SB}=\mathrm{Ca}^{2+}+\mathrm{Mg}^{2+}+\mathrm{K}^{+}+\mathrm{Na}^{+}\right)$, equivalent cation exchange capacity $\left(E C E C=\mathrm{SB}+\mathrm{Al}^{3+}\right)$, total cation exchange capacity $[C E C=S B+(H+A l)]$ base saturation $(V=S B / C E C)$ and aluminum saturation $(m=$ $\mathrm{Al}^{3+} / \mathrm{ECEC}$ ) were calculated. A Mehlich-1 extraction solution determined the soil $\mathrm{P}$ status for plant growth $\left(\mathrm{P}_{\mathrm{M}}\right)$. Total organic carbon $(\mathrm{C})$ was determined by Walkey and Black method (YEOMANS and BREMNER, 1988). Total nitrogen $(\mathrm{N})$ was determined by the Kjeldahl method and titration (EMBRAPA, 1997). The carbon to nitrogen ratio $(\mathrm{C} / \mathrm{N})$ was calculated on a mass basis. The $P$ adsorption capacity of the soil was determined after stirring it for 1 hour with $2.5 \mathrm{~g}$ of soil in $0.01 \mathrm{M}$ $\mathrm{CaCl}_{2}$ containing $60 \mathrm{mg}$ of $\mathrm{P} \mathrm{L}^{-1}$. The suspension was stirred for $5 \mathrm{~min}$ on a helical motion shaker and left to stand for 16 hours, and the remaining $P$ in solution ( $P_{\text {REM }}$ ) was determined by photocolorimetry (ALVAREZ et al., 2000).

Bulk density, particle density and water retention curve $(-6,-10,-30,-60,-100,-300,-1500 \mathrm{kPa}$ tensions) were determined in undisturbed soil samples collected by volumetric rings. Total porosity and available water were calculated from these results. The tension of $-1500 \mathrm{kPa}$ was considered the wilting point.

\section{RESULTS}

We have identified different soil groups in semideciduous and dry forests. Four of the seven collected soil profiles in the semideciduous forest were classified as Umbrisols according to WRB and as Neossolos Regolíticos in the Brazilian Soil Classification System. Three Stagnosols were described in a concave slope: a Pantoorthodystric Stagnosols with non-cemented iron and manganese masses (Gleissolo Háplico) in the upper slope; a Pantoorthodystric Anoumbric Stagnosols (Gleissolo Melânico) with non-cemented iron and manganese masses in the midslope; and a Pantoorthodystric Anoumbric Stagnosols (Gleissolo Melânico) in the base slope (Table 1). In general, soils are composed by a sequence of $A$ and $C$ horizons. Tree Luvisols (Luvissolos Crômicos) and one Regosol (Neossolo Regolítico) were described on a partially dissected pavement covered by dry forest.

The depth of the soils in semideciduous forest varied between $55 \mathrm{~cm}$ and $160 \mathrm{~cm}$ with a mean of 117 $\mathrm{cm}$ (Table 2). Soils are deep, and the transition between horizons was predominantly gradual, indicating high homogeneity between horizons. Soils in the dry forest are slightly shallower than the ones in the semideciduous forest.

The structure is low to moderately developed, with subangular blocky and granular types dominantly. Wormholes and sand-filled animal burrows were observed in all Regosols, indicating widespread bioturbation. Roots are denser in shallow horizons, and their growth is limited by lithic contact. Medium and coarse roots occur below $70 \mathrm{~cm}$ of depth. The soil color ranged from greenish-black in horizon $\mathrm{C}$, with stagnic properties, to dark reddish gray in umbric epipedon. The umbric epipedon described in the soil profiles indicates melanization.

The texture of semideciduous and dry forest soils is similar. The soil profiles vary between loamy sand and clay. In general, a slight increase in clay content in deeper horizons is observed (Table 3). Coarse sand is the main fraction of soil, once its average content is approximately $40 \%$. The clay content in subsurface horizons increases with the increase of altitude; the subsurface horizon of soils in the upper slope has an average clay content even nine times higher than the other ones. Quartz was identified in the field as the main component of coarse fraction in all soils. 
Table 1 - Borborema province: sites description and classification of soils.

\section{Soil WRB}

profile

Semideciduous forest soils

1

SiBCS

Altitude Area description

(m)
(Endoclayic,

Hyperdystric)

NEOSSOLO

REGOLÍTICO

Húmico léptico

2

(Pantoloamic,

Pantohyperdystric)

NEOSSOLO

REGOLÍTICO

3

Pantoorthodystric

Stagnosols

(Amphiclayic,

Katomanganiferric

Humic, Inclinic)

4

Pantoorthodystric

Anoumbric

Stagnosols

(Pantoclayic, Humic,

Inclinic)

$5 \quad$ Leptic Umbrisol

(Endoclayic,

Pantodystric)
Haplic Umbrisol

Húmico típico
GLEISSOLO

HÁPLICO Tb

Distrófico

argissólico

GLEISSOLO

MELÂNICO Tb

Distrófico típico

640

NEOSSOLO

REGOLÍTICO

Húmico léptico

650

So

Soil developed on the shoulder of rock outcrop. Well-drained soils, with no apparent eros
and derived from granitoid colluvium. Soil is covered by litter and subperennial arboreal
specimens. Fabaceae, Myrtaceae, Sapindaceae families, and Allophylus puberulus

(Cambess.) Radlk., Campomanesia aromatica (Aubl.) Griseb., Cupania impressinervia

Acev.-Rodr., Guapira opposita (Vell.) Reitz species dominate. Clay texture, umbric

epipedon, and gentle slope surface.

641 Soil developed on footslope of rock outcrop. Well-drained soils, with no apparent erosion and derived from granitoid colluvium. Soil is covered by litter and subperennial arboreal specimens. Myrtaceae e Salicaceae families and Aspidosperma parvifolium A.DC. Handroanthus serratifolius (Vahl), Zanthoxylum petiolare A. St.-Hil. \& Tul. Species dominate. Sandy clay loam texture, umbric epipedon and gentle slope surface.

657 Soil developed on summit of rock outcrop. Poorly drained soils, with no apparent erosion and derived from granitoid colluvium. Soil is covered by litter and subperennial arboreal specimens. Fabaceae and Myrtaceae families and Byrsonima vacciniifolia A.Juss., Guapira opposita (Vell.) Reitz., Myrcia splendens (Sw.) DC. Species dominate. Sandy clay texture, umbric epipedon and gentle slope surface.

Soil developed on footslope of rock outcrop. Poorly drained soils, with no apparent erosion and derived from granitoid colluvium. Soil is covered by litter and subperennial arboreal specimens. Fabaceae and Myrtaceae families and Allophylus puberulus (Cambess.) Radlk. Casearia aculeata Jacq., Cynophalla flexuosa (L.) J.Presl, Zanthoxylum petiolare A. St.-Hil. $\&$ Tul. Species dominate. Loamy sand texture, umbric epipedon and gentle slope surface.

Soil developed on shoulder of rock outcrop. Well drained soils, with no apparent erosion and derived from granitoid colluvium. Soil is covered by litter and subperennial arboreal specimens. Bignoniaceae, Fabaceae, Myrtaceae, Cordiaceae families and Allophylus puberulus (Cambess.) Radlk., Casearia aculeata Jacq., Manilkara salzmannii (A. DC.) H. J. Lam. Species dominate. Sandy clay texture, umbric epipedon and gentle slope surface. 
$6 \quad$ Pantoorthodystric

Anoumbric

Stagnosols

(Pantoclayic,

Katomanganiferric,

Humic, Inclinic)

7

Leptic Umbrisol

(Pantoloamic,

Hyperdystric)
GLEISSOLO

MELÂNICO Tb

Distrófico típico

NEOSSOLO

REGOLÍTICO

Distrófico léptico
636

Soil developed on footslope of rock outcrop. Poorly drained soils, with no apparent erosion and derived from granitoid colluvium. Soil is covered by litter and subperennial arboreal specimens. Euphorbiaceae e Myrtaceae families and Allophylus puberulus (Cambess.) Radlk., Psidium oligospermum Mart. ex DC., Sebastiania jacobinensis (Müll.Arg.) Müll.Arg. species dominate. Loamy sand texture, umbric epipedon and gentle slope surface.

Soil developed on backslope of rock outcrop. Well drained soils, with no apparent erosion and derived from granitoid colluvium. Soil is covered by litter and subperennial arboreal specimens. Fabaceae, Myrtaceae, Sapindaceae families and Bowdichia virgilioides Kunth, Campomanesia dichotoma (O. Berg) Mattos, Cupania impressinervia Acev.-Rodr. species dominate. Sandy loam texture, umbric epipedon and gentle slope surface. $-6.831324^{\circ}$

$-35.614601^{\circ}$

$-6.648407^{\circ}$

$-35.610948^{\circ}$

Dry forest soils

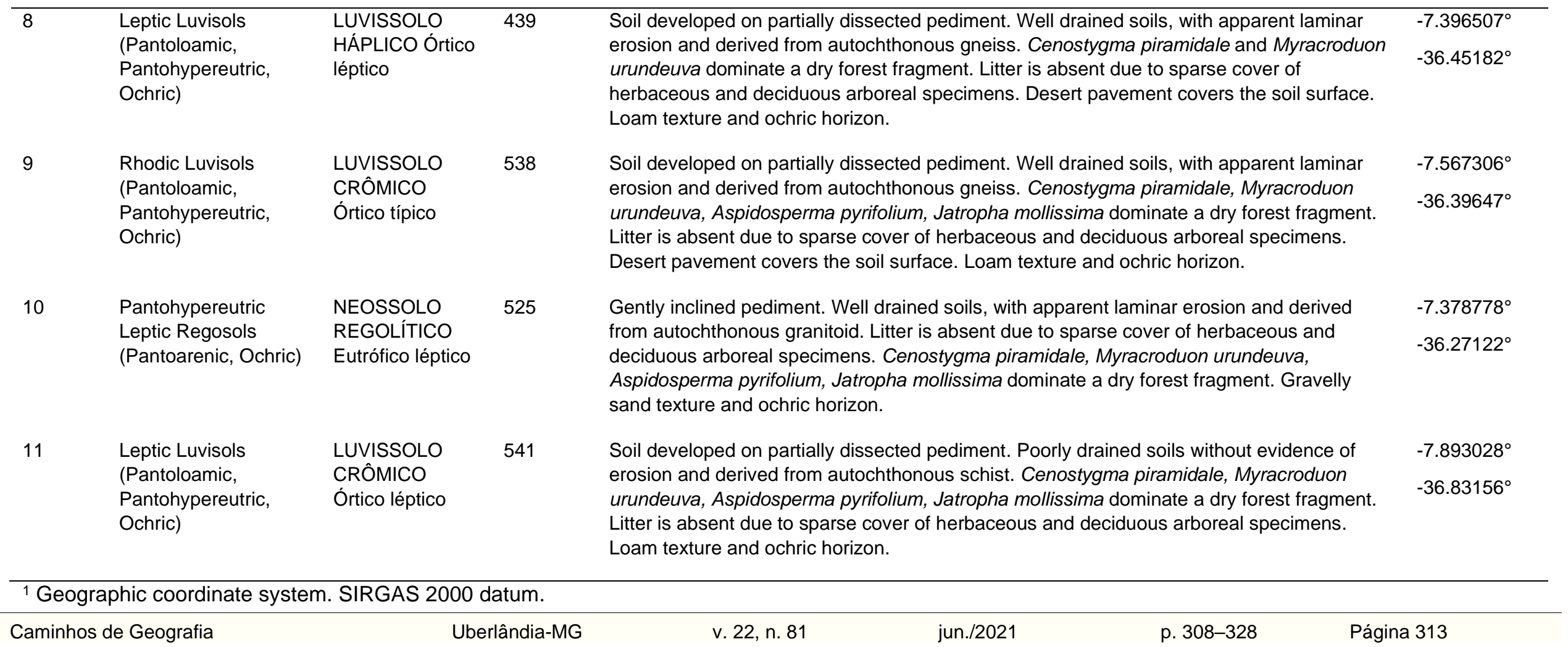


Table 2 - Borborema province: Morphological properties of soils.

\begin{tabular}{|c|c|c|c|c|c|c|c|c|c|}
\hline $\begin{array}{l}\text { Horiz } \\
\text { on }\end{array}$ & $\begin{array}{l}\text { Dep } \\
\text { th } \\
(\mathrm{cm})\end{array}$ & $\begin{array}{l}\text { Boundary } \\
\text { (distinctn } \\
\text { ess, } \\
\text { topograp } \\
\text { hy) }\end{array}$ & $\begin{array}{l}\text { Moist } \\
\text { color }\end{array}$ & $\begin{array}{l}\text { Struct } \\
\text { ure } \\
\text { (grade, } \\
\text { size, } \\
\text { type) }\end{array}$ & $\begin{array}{l}\text { Consiste } \\
\text { nce (dry, } \\
\text { moist, } \\
\text { wetter, } \\
\text { cementati } \\
\text { on) }\end{array}$ & $\begin{array}{l}\text { Redoximor } \\
\text { phic } \\
\text { features } \\
\text { (kind, } \\
\text { quantity, } \\
\text { size, } \\
\text { contrast, } \\
\text { state, } \\
\text { hardness, } \\
\text { boundary) }\end{array}$ & $\begin{array}{l}\text { Redoximor } \\
\text { phic color } \\
\text { (matrix, } \\
\text { masses) }\end{array}$ & $\begin{array}{l}\text { Roots } \\
\text { (quanti } \\
\text { ty, } \\
\text { size) }\end{array}$ & $\begin{array}{l}\text { Pores } \\
\text { (quanti } \\
\text { ty, } \\
\text { size, } \\
\text { shape) }\end{array}$ \\
\hline
\end{tabular}

\begin{tabular}{|c|c|c|c|c|c|c|c|}
\hline \multicolumn{8}{|c|}{ Semideciduous forest soils } \\
\hline \multicolumn{8}{|c|}{ Leptic Umbrisol (Endoclayic, Hyperdystric)/ NEOSSOLO REGOLÍTICO Húmico léptico } \\
\hline $\mathrm{A} 1$ & $0-20$ & G-S & $\begin{array}{l}10 \mathrm{YR} \\
2 / 1\end{array}$ & $\begin{array}{l}2, \quad m, \\
\text { sbk }\end{array}$ & $\begin{array}{l}\text { SH, } \quad F R, \\
\text { ss, ps, NC }\end{array}$ & $\begin{array}{l}2, \quad c o- \\
m-f\end{array}$ & $\begin{array}{l}2, \quad \text { co- } \\
m-f, \text { DT }\end{array}$ \\
\hline $\mathrm{A} 2$ & $\begin{array}{l}20- \\
30\end{array}$ & G-S & $\begin{array}{l}10 Y R \\
2 / 1\end{array}$ & $\begin{array}{l}2, \quad m, \\
\text { sbk }\end{array}$ & $\begin{array}{l}\text { SH, FR, } \\
\text { ss, vp, NC }\end{array}$ & $\begin{array}{l}2, \quad m-f- \\
v f\end{array}$ & $2, \mathrm{f}, \mathrm{DT}$ \\
\hline$A C$ & $\begin{array}{l}30- \\
50\end{array}$ & G-S & $\begin{array}{l}7.5 Y R \\
2.6 / 1\end{array}$ & $\begin{array}{l}2, \quad \mathrm{~m}, \\
\text { sbk }\end{array}$ & $\begin{array}{ll}\mathrm{SH}, & \mathrm{FR}, \\
\mathrm{ms}, & \mathrm{vp}, \\
\mathrm{NC} & \end{array}$ & $2, m-f$ & $\begin{array}{l}\text { 2, f-vf, } \\
\text { DT }\end{array}$ \\
\hline $\mathrm{Cr}$ & $\begin{array}{l}50- \\
55\end{array}$ & C-S & $\begin{array}{l}2.5 Y R \\
2.5 / 4\end{array}$ & $0,-, m$ & $\begin{array}{ll}\mathrm{SH}, & \mathrm{FR}, \\
\mathrm{ms}, & \mathrm{ps}, \\
\mathrm{NC} & \end{array}$ & absent & $1, f, I G$ \\
\hline
\end{tabular}

R $\quad 55+$

Haplic Umbrisol (Pantoloamic, Pantohyperdystric)/ NEOSSOLO REGOLÍTICO Húmico típico

\begin{tabular}{|c|c|c|c|c|c|c|c|}
\hline A1 & $0-40$ & G-S & $\begin{array}{l}10 \mathrm{YR} \\
2 / 1\end{array}$ & $2, \mathrm{f}, \mathrm{gr}$ & $\begin{array}{l}\text { SH, FR, } \\
\text { ss, ps, NC }\end{array}$ & $\begin{array}{l}3, \quad m-f- \\
v f\end{array}$ & $\begin{array}{l}2, \quad m-f \\
\text { DT }\end{array}$ \\
\hline $\mathrm{A} 2$ & $\begin{array}{l}40- \\
62\end{array}$ & G-S & $\begin{array}{l}7.5 Y R \\
2.5 / 1\end{array}$ & $1, \mathrm{f}, \mathrm{gr}$ & $\begin{array}{l}\text { SH, } \quad F R, \\
\text { ss, ps, NC }\end{array}$ & $\begin{array}{l}2, m-f- \\
\text { vf }\end{array}$ & $\begin{array}{l}\text { 2, f-vf, } \\
\text { DT }\end{array}$ \\
\hline A3 & $\begin{array}{l}62- \\
100\end{array}$ & C-S & $\begin{array}{l}2.5 Y R \\
2.5 / 1\end{array}$ & $\begin{array}{l}2, \quad m, \\
\text { sbk }\end{array}$ & $\begin{array}{ll}\mathrm{SH}, & \mathrm{FR}, \\
\mathrm{sS}, & \mathrm{mp}, \\
\mathrm{NC} & \end{array}$ & $2, \mathrm{f}-\mathrm{vf}$ & $2, \mathrm{f}, \mathrm{DT}$ \\
\hline$A C$ & $\begin{array}{l}100- \\
140\end{array}$ & A-S & $\begin{array}{l}5 Y R \\
3 / 3\end{array}$ & $\begin{array}{l}1, \\
\text { sbk }\end{array}$ & $\begin{array}{l}\mathrm{SH}, \quad \mathrm{FR}, \\
\text { ss, ps, NC }\end{array}$ & $1, f-v f$ & $2, \mathrm{f}, \mathrm{DT}$ \\
\hline$R$ & $\begin{array}{l}140 \\
+\end{array}$ & & & & & & \\
\hline
\end{tabular}

Pantoorthodystric Stagnosols (Amphiclayic, Katomanganiferric, Humic, Inclinic)/ GLEISSOLO HÁPLICO Tb Distrófico argissólico

\begin{tabular}{|c|c|c|c|c|c|c|c|c|c|c|}
\hline A & $0-25$ & G-S & $\begin{array}{l}2.5 \mathrm{YR} \\
3 / 1\end{array}$ & $\begin{array}{l}1, \\
\text { sbk }\end{array}$ & $\mathrm{m}$, & $\begin{array}{l}\mathrm{SH}, \quad \mathrm{FR}, \\
\text { so, po, } \mathrm{NC}\end{array}$ & & & $\begin{array}{l}2, \quad m- \\
\text { co-f-vf }\end{array}$ & $\begin{array}{l}2, \\
\text { TU }\end{array}$ \\
\hline$A B$ & $\begin{array}{l}25- \\
60\end{array}$ & G-S & $\begin{array}{l}5 Y R \\
3 / 1\end{array}$ & $\begin{array}{l}2, \\
\text { sbk }\end{array}$ & $\mathrm{m}$, & $\begin{array}{l}\text { SH, } \quad F R, \\
\text { ss, ps, NC }\end{array}$ & & & $1, m, f$ & $\begin{array}{l}\text { 2, } \\
\text { TU }\end{array}$ \\
\hline Btg & $\begin{array}{l}70- \\
90(6 \\
0- \\
100)\end{array}$ & $C-W$ & $\begin{array}{l}\text { GLEY1 } \\
4 / 10 Y\end{array}$ & $\begin{array}{l}2, \\
\text { sbk }\end{array}$ & $\mathrm{m}$, & $\begin{array}{l}\text { SH, } \quad F R, \\
\text { vs, vp, NC }\end{array}$ & $\begin{array}{l}\text { FMM, f, 1, } \\
\text { D, M, VW, } \\
\text { C }\end{array}$ & $\begin{array}{l}\text { GLEY1 } \\
4 / 10 Y, 5 Y R \\
3 / 2\end{array}$ & $1, \mathrm{v}-\mathrm{vf}$ & $\begin{array}{l}2,{ }^{m-} \\
\text { TU }\end{array}$ \\
\hline Cgr & $\begin{array}{l}90- \\
150 \\
+\end{array}$ & & $\begin{array}{l}\text { GLEY1 } \\
2.5 / \mathrm{N}\end{array}$ & $\begin{array}{l}1, \\
\text { sbk }\end{array}$ & $\mathrm{m}$, & $\begin{array}{l}\text { SH, FR, } \\
\text { vs, vp, NC }\end{array}$ & $\begin{array}{l}\text { FMM, m, 1, } \\
\text { D, M, VW, } \\
\text { C }\end{array}$ & $\begin{array}{l}\text { GLEY1 } \\
2.5 / \mathrm{N}, \quad 10 \mathrm{R} \\
4 / 8\end{array}$ & absent & $1, \mathrm{f}, \mathrm{TU}$ \\
\hline
\end{tabular}

Pantoorthodystric Anoumbric Stagnosols (Pantoclayic, Humic, Inclinic)/ GLEISSOLO MELÂNICO Tb Distrófico típico
Ag $\quad 0-70 \quad$ G-S
GLEY1
2, $\mathrm{m}, \mathrm{SH}, \quad \mathrm{FR}$,
3, co- 2, m,

Caminhos de Geografia

Uberlândia-MG

v. 22, n. $81 \quad$ jun./2021

p. 308-328

Página 314 


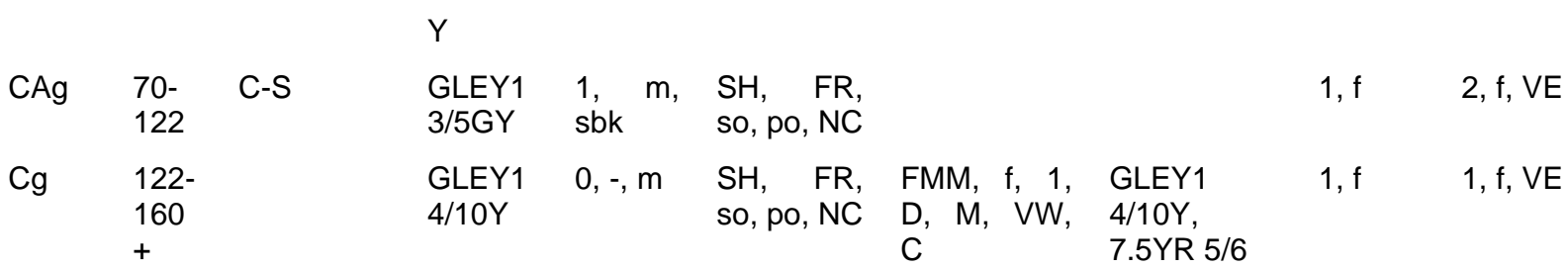

Leptic Umbrisol (Endoclayic, Pantodystric)/ NEOSSOLO REGOLÍTICO Húmico léptico

\begin{tabular}{|c|c|c|c|c|c|c|c|}
\hline A1 & $0-27$ & G-S & $\begin{array}{l}2.5 Y \\
3 / 1\end{array}$ & $\begin{array}{l}2, \quad \mathrm{~m}, \\
\mathrm{gr}\end{array}$ & $\begin{array}{l}\text { SH, FR, } \\
\text { ss, ps, NC }\end{array}$ & $\begin{array}{l}2, \quad m- \\
c o-f\end{array}$ & $\begin{array}{l}2, \quad m, \\
\text { TU }\end{array}$ \\
\hline A2 & $\begin{array}{l}27- \\
47\end{array}$ & C-S & $\begin{array}{l}7.5 \mathrm{YR} \\
3 / 2\end{array}$ & $\begin{array}{l}2, \quad \mathrm{~m} / \mathrm{f} \\
\mathrm{sbk} / \mathrm{gr}\end{array}$ & $\begin{array}{l}\mathrm{SH} \\
\mathrm{ms}, \\
\mathrm{NC}\end{array}$ & $\begin{array}{l}1, \quad v c- \\
\text { co-m-f }\end{array}$ & $\begin{array}{l}1, \\
\text { TU }\end{array}$ \\
\hline $\mathrm{Cr}$ & $\begin{array}{l}47- \\
67\end{array}$ & & $\begin{array}{l}\text { variegat } \\
\text { ed }\end{array}$ & $0,-, \mathrm{m}$ & $\begin{array}{l}\text { SH, FR, } \\
\text { so, po, NC }\end{array}$ & $1, f$ & $1, f, I G$ \\
\hline
\end{tabular}

R $67+$

Pantoorthodystric Anoumbric Stagnosols (Pantoclayic, Katomanganiferric, Humic, Inclinic)/GLEISSOLO MELÂNICO Tb Distrófico típico

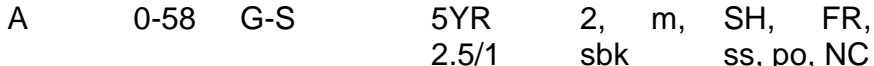
2 , co- 2, m,

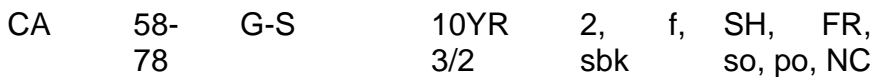
2, f-vf $1, \mathrm{f}, \mathrm{IG}$
Cg $\quad 78-$
GLEY1 2, m, SH, FR, FMM, f, 1, GLEY1
$5 / 10 Y$ sbk
so, po, NC
D, M, VW, 5/10Y,
$2, \mathrm{f}-\mathrm{vf} \quad 1, \mathrm{f}, \mathrm{IG}$
$+\quad$ C $\quad 7.5$ YR 5/6

Leptic Umbrisol (Pantoloamic, Hyperdystric)/ NEOSSOLO REGOLÍTICO Distrófico léptico

\begin{tabular}{|c|c|c|c|c|c|c|c|}
\hline A & $0-45$ & C-S & $\begin{array}{l}7.5 \mathrm{YR} \\
3 / 1\end{array}$ & $\begin{array}{l}1, \quad \mathrm{~m}, \\
\mathrm{sbk}\end{array}$ & $\begin{array}{l}\text { SH, FR, } \\
\text { so, po, NC }\end{array}$ & $\begin{array}{l}3, \quad \mathrm{co}- \\
\mathrm{m}-\mathrm{f}\end{array}$ & $\begin{array}{l}2, \quad \mathrm{co}- \\
\mathrm{m}, \mathrm{TU}\end{array}$ \\
\hline$A C$ & $\begin{array}{l}45- \\
100\end{array}$ & A-S & $\begin{array}{l}5 Y R \\
3 / 2\end{array}$ & $\begin{array}{l}2, \quad m, \\
\text { sbk }\end{array}$ & $\begin{array}{l}\text { SH, FR, } \\
\text { so, po, NC }\end{array}$ & $\begin{array}{l}3, m-f- \\
\text { vf }\end{array}$ & $2, \mathrm{f}, \mathrm{TU}$ \\
\hline $\mathrm{R}$ & $\begin{array}{l}100 \\
+\end{array}$ & & & & & & \\
\hline \multicolumn{8}{|c|}{ Dry forest soils } \\
\hline \multicolumn{8}{|c|}{ Leptic Luvisols (Pantoloamic, Pantohypereutric, Ochric)/ LUVISSOLO HÁPLICO Órtico léptico } \\
\hline A & $0-10$ & C-S & $10 \mathrm{R} 3 / 4$ & $\begin{array}{l}2, \\
\text { sbk }\end{array}$ & $\begin{array}{l}\mathrm{SH}, \quad \mathrm{FR}, \\
\mathrm{vs}, \mathrm{p}, \mathrm{NC}\end{array}$ & $\begin{array}{l}1, f-v f- \\
m\end{array}$ & $\begin{array}{l}3, \quad f-v f- \\
m, \text { DT }\end{array}$ \\
\hline $\mathrm{Bt}$ & $\begin{array}{l}10- \\
30\end{array}$ & C-S & $\begin{array}{ll}2.5 & \text { YR } \\
3 / 2 & \end{array}$ & $\begin{array}{l}2, \quad \mathrm{~m}, \\
\text { sbk }\end{array}$ & $\begin{array}{l}\mathrm{H}, \mathrm{FI}, \mathrm{vs}, \\
\mathrm{vp}, \mathrm{NC}\end{array}$ & $\begin{array}{l}3, \quad f-v f- \\
m\end{array}$ & $\begin{array}{l}1, \mathrm{f}-\mathrm{vf}- \\
\mathrm{m}, \mathrm{TU}\end{array}$ \\
\hline CR & $\begin{array}{l}30- \\
50\end{array}$ & C-S & $\begin{array}{l}7.5 Y R \\
4 / 4\end{array}$ & $0,-, m$ & $\begin{array}{l}\mathrm{H}, \mathrm{FI}, \mathrm{ms} \\
\mathrm{mp}, \mathrm{NC}\end{array}$ & absent & $\begin{array}{l}2, \\
\text { VE }\end{array}$ \\
\hline
\end{tabular}

R $50+$

Rhodic Luvisols (Pantoloamic, Pantohypereutric, Ochric)/ LUVISSOLO CRÔMICO Órtico típico

\begin{tabular}{|c|c|c|c|c|c|c|c|}
\hline A & $\begin{array}{l}0-3 \\
(2-5)\end{array}$ & C-W & $\begin{array}{l}7.5 Y R \\
3 / 3\end{array}$ & $\begin{array}{l}2, \\
\text { sbk }\end{array}$ & $\begin{array}{l}\mathrm{H}, \mathrm{FR} \text {, so, } \\
\text { po, NC }\end{array}$ & $1, \mathrm{vf}$ & $2, \mathrm{f}, \mathrm{TU}$ \\
\hline Bt1 & $3-25$ & G-S & $\begin{array}{l}7.5 Y R \\
3 / 4\end{array}$ & $\begin{array}{l}1, \quad m, \\
\text { sbk }\end{array}$ & $\begin{array}{l}\mathrm{H}, \mathrm{FI}, \mathrm{vs}, \\
\mathrm{mp}, \mathrm{NC}\end{array}$ & $2, f-v f$ & $\begin{array}{l}3, \\
\text { TU }\end{array}$ \\
\hline Bt2 & $\begin{array}{l}25- \\
50\end{array}$ & C-S & $\begin{array}{l}7.5 Y R \\
3 / 6\end{array}$ & $\begin{array}{l}\text { 3, m, } \\
\mathrm{pr}\end{array}$ & $\begin{array}{l}\mathrm{H}, \mathrm{FI}, \mathrm{vs}, \\
\mathrm{vp}, \mathrm{NC}\end{array}$ & $1, f-v f$ & $\begin{array}{l}2, \\
I G\end{array}$ \\
\hline $\mathrm{Cr}$ & $\begin{array}{l}50- \\
75\end{array}$ & G-S & $\begin{array}{l}7.5 Y R \\
5 / 6\end{array}$ & $0,-, \mathrm{m}$ & $\begin{array}{l}\mathrm{H}, \mathrm{FI}, \mathrm{ms} \\
\mathrm{mp}, \mathrm{NC}\end{array}$ & absent & $\begin{array}{l}2, \\
\text { VE }\end{array}$ \\
\hline
\end{tabular}


R 75+

Pantohypereutric Leptic Regosols (Pantoarenic, Ochric)/ NEOSSOLO REGOLÍTICO Eutrófico léptico

\begin{tabular}{|c|c|c|c|c|c|c|c|}
\hline A & $0-5$ & C-S & $\begin{array}{l}10 Y R \\
2 / 2\end{array}$ & $\begin{array}{l}2, \quad m, \\
\text { sbk }\end{array}$ & $\begin{array}{l}\text { L, FR, so, } \\
\text { po, NC }\end{array}$ & $2, \mathrm{f}-\mathrm{vf}$ & $\begin{array}{l}3, \quad m-f, \\
\text { TU }\end{array}$ \\
\hline AC1 & $5-15$ & C-S & $\begin{array}{l}7.5 Y R \\
3 / 3\end{array}$ & $\begin{array}{l}2, \quad \mathrm{~m}, \\
\mathrm{sbk}\end{array}$ & $\begin{array}{l}\text { S, FR, so, } \\
\text { po, NC }\end{array}$ & $2, \mathrm{f}-\mathrm{vf}$ & $2, \mathrm{f}, \mathrm{TU}$ \\
\hline AC2 & $\begin{array}{l}15- \\
30\end{array}$ & C-S & $\begin{array}{l}7.5 Y R \\
4 / 4\end{array}$ & $\begin{array}{l}2, \quad m, \\
\text { sbk }\end{array}$ & $\begin{array}{l}\text { SH, FR, } \\
\text { so, po, NC }\end{array}$ & $\begin{array}{l}2, \quad \mathrm{co}- \\
\mathrm{m}-\mathrm{f}\end{array}$ & $\begin{array}{l}2, \quad f-1 \\
T U\end{array}$ \\
\hline C & $\begin{array}{l}30- \\
60\end{array}$ & $A-S$ & $\begin{array}{l}5 Y R \\
4 / 4\end{array}$ & $\begin{array}{l}1, \quad f, \\
\text { sbk }\end{array}$ & $\begin{array}{l}\text { S, L, so, } \\
\text { po, NC }\end{array}$ & $1, \mathrm{vf}$ & $\begin{array}{l}2, \quad f- \\
I G\end{array}$ \\
\hline
\end{tabular}

R $60+$

Leptic Luvisols (Pantoloamic, Pantohypereutric, Ochric)/ LUVISSOLO CRÔMICO Órtico léptico

\begin{tabular}{|c|c|c|c|c|c|c|}
\hline A & $0-5$ & C-S & $\begin{array}{l}5 \mathrm{YR} \\
4 / 2\end{array}$ & $\begin{array}{l}2, \quad m, \\
\text { sbk }\end{array}$ & $\begin{array}{l}\text { SH, FR, } \\
\text { ss, ps, NC }\end{array}$ & $2, f$ \\
\hline $\mathrm{Bt}$ & $5-25$ & C-S & $\begin{array}{l}5 \mathrm{YR} \\
3 / 4\end{array}$ & $\begin{array}{l}\text { 2, } \mathrm{m}, \\
\mathrm{pr}\end{array}$ & $\begin{array}{l}\mathrm{H}, \mathrm{FR}, \mathrm{s}, \\
\mathrm{ps}, \mathrm{NC}\end{array}$ & $2, \mathrm{~m}$ \\
\hline C & $\begin{array}{l}25- \\
55\end{array}$ & C-S & $\begin{array}{l}7.5 Y R \\
4 / 4\end{array}$ & $2, \mathrm{f}, \mathrm{pr}$ & $\begin{array}{l}\text { VH, FI, vs, } \\
\text { vp, NC }\end{array}$ & $1, \mathrm{~m}$ \\
\hline
\end{tabular}

R $55+$

Distinctness: $\mathrm{A}=$ abrupt, $\mathrm{C}=$ clear, $\mathrm{G}=$ gradual. Topography: $\mathrm{S}=$ smooth, $\mathrm{W}=$ wavy. Structure: Grade: $0=$ structureless, 1 =weak, $2=$ moderate. Size: $\mathrm{f}=$ fine, $\mathrm{m}=$ medium. Type: $g r=$ granular, $\mathrm{m}=\mathrm{massive}, \mathrm{sbk}=$ subangular block, /=principal structure parting to secondary one. Consistence: Dry: L=loose, $\mathrm{S}=\mathrm{Soft}$, $\mathrm{SH}=$ slightly hard. Moist: $\mathrm{Fl}=$ firm, $\mathrm{FR}=$ friable, $\mathrm{L}=$ loose. Wetter: $\mathrm{ms}=$ moderately stick, vs=very stick, $\mathrm{so}=$ nonsticky, $\mathrm{ss}=$ slightly sticky, $\mathrm{mp}=$ moderately plastic, $\mathrm{po}=$ nonplastic, $\mathrm{ps}=$ slightly plastic, $\mathrm{vp}=\mathrm{very}$ plastic. Cementation: $\mathrm{NC}=$ non-cemented. Redoximorphic features: Kind: $\mathrm{FMM}=$ non-cemented ironmanganese masses. Quantity: $f=f e w, m=m a n y$. Size: $1=f i n e$. Contrast: $D=d i s t i n c t$. State: $M=m o i s t$. Hardness: VW=very weakly cemented. Boundary: $C=$ clear. Roots and pores: $1=$ few, $2=$ common, $3=$ many, $c o=$ coarse, $f=$ fine, $m=$ medium, $v c=$ very coarse, $v f=v e r y$ fine, $D T=d e n d r i t i c$ tubular, $\mathrm{IG}=$ irregular, $\mathrm{TU}=$ tubular, $\mathrm{VE}=$ vesicular.

Table 3 - Borborema province: Physical soil data of soil profiles and comparison data.

\begin{tabular}{|c|c|c|c|c|c|c|}
\hline Horizon & $\begin{array}{l}\text { Depth } \\
\text { cm }\end{array}$ & Coarse sand & $\begin{array}{l}\text { Fine sand } \\
---\% ~\end{array}$ & Silt & Clay & Texture \\
\hline \multicolumn{7}{|c|}{ Semideciduous forest soils } \\
\hline \multicolumn{7}{|c|}{ Leptic Umbrisol (Endoclayic, Hyperdystric)/ NEOSSOLO REGOLÍTICO Húmico léptico } \\
\hline A1 & $0-20$ & 28.1 & 20.9 & 14.1 & 36.8 & sandy clay \\
\hline A2 & $20-30$ & 32.4 & 18.7 & 13.2 & 35.7 & sandy clay \\
\hline AC & $30-50$ & 17.8 & 11.2 & 10.5 & 60.5 & clay \\
\hline $\mathrm{Cr}$ & $50-55$ & 14.3 & 6.5 & 10.1 & 69.1 & clay \\
\hline $\mathrm{R}$ & $55+$ & & & & & \\
\hline \multicolumn{7}{|c|}{ Haplic Umbrisol (Pantoloamic, Pantohyperdystric)/ NEOSSOLO REGOLÍTICO Húmico típico } \\
\hline A1 & $0-40$ & 37.6 & 18.3 & 15.8 & 28.4 & sandy clay loam \\
\hline A2 & $40-62$ & 36.0 & 21.8 & 15.5 & 26.6 & sandy clay loam \\
\hline A3 & $62-100$ & 31.9 & 23.8 & 15.8 & 28.6 & sandy clay loam \\
\hline$A C$ & $100-140$ & 33.1 & 17.8 & 16.9 & 32.2 & sandy clay loam \\
\hline
\end{tabular}


Pantoorthodystric Stagnosols (Amphiclayic, Katomanganiferric, Humic, Inclinic)/ GLEISSOLO HÁPLICO Tb Distrófico argissólico

$\begin{array}{lllllll}\text { A } & 0-25 & 40.0 & 31.5 & 7.8 & 20.7 & \text { sandy clay loam } \\ \text { AB } & 25-60 & 38.2 & 27.8 & 11.1 & 22.9 & \text { sandy clay loam } \\ \text { Btg } & 70-90(60-100) & 25.3 & 22.0 & 11.3 & 41.4 & \text { sandy clay } \\ \text { Cgr } & 90-150+ & 21.5 & 11.7 & 10.7 & 56.2 & \text { clay }\end{array}$

Pantoorthodystric Anoumbric Stagnosols (Pantoclayic, Humic, Inclinic)/ GLEISSOLO MELÂNICO Tb Distrófico típico

$\begin{array}{lllllll}\mathrm{Ag} & 0-70 & 51.1 & 21.5 & 13.3 & 14.2 & \text { sandy loam } \\ \mathrm{CAg} & 70-122 & 60.0 & 22.9 & 11.6 & 5.5 & \text { loamy sand } \\ \mathrm{Cg} & 122-160+ & 59.1 & 24.4 & 10.9 & 5.5 & \text { loamy sand }\end{array}$

Leptic Umbrisol (Endoclayic, Pantodystric)/ NEOSSOLO REGOLÍTICO Húmico léptico

$\begin{array}{lllllll}\text { A1 } & 0-27 & 51.8 & 13.1 & 6.3 & 28.8 & \text { sandy clay loam } \\ \text { A2 } & 27-47 & 47.5 & 11.7 & 12.0 & 28.8 & \text { sandy clay loam } \\ \mathrm{Cr} & 47-67 & 42.5 & 10.2 & 10.3 & 37.0 & \text { sandy clay }\end{array}$

R $67+$

Pantoorthodystric Anoumbric Stagnosols (Pantoclayic, Katomanganiferric, Humic, Inclinic)/GLEISSOLO MELÂNICO Tb Distrófico típico

$\begin{array}{lllllll}\text { A } & 0-58 & 45.1 & 26.1 & 14.0 & 14.9 & \text { sandy loam } \\ \mathrm{CA} & 58-78 & 51.0 & 23.7 & 17.4 & 8.0 & \text { sandy loam } \\ \mathrm{Cg} & 78-150+ & 52.5 & 25.6 & 14.3 & 7.6 & \text { loamy sand }\end{array}$

Leptic Umbrisol (Pantoloamic, Hyperdystric)/ NEOSSOLO REGOLÍTICO Distrófico léptico

$\begin{array}{lllllll}\text { A } & 0-45 & 62.3 & 19.0 & 8.2 & 10.5 & \text { loamy sand } \\ \text { AC } & 45-100 & 50.4 & 22.4 & 16.5 & 10.7 & \text { sandy loam } \\ \text { R } & 100+ & & & & & \end{array}$

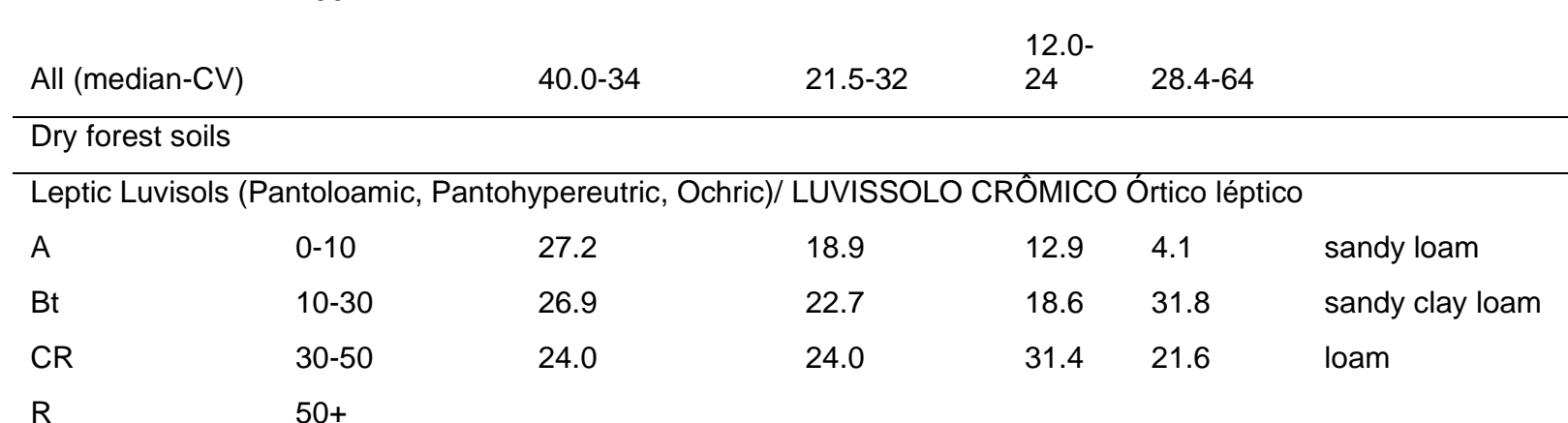

Rhodic Luvisols (Pantoloamic, Pantohypereutric, Ochric)/ LUVISSOLO CRÔMICO Órtico típico

$\begin{array}{lllllll}\text { A } & 0-3(2-5) & 30.9 & 19.4 & 24.5 & 25.3 & \text { sandy clay loam } \\ \text { Bt1 } & 3-25 & 26.3 & 12.8 & 26.8 & 34.0 & \text { sandy clay loam } \\ \text { Bt2 } & 25-50 & 27.7 & 15.5 & 27.2 & 29.6 & \text { sandy clay loam } \\ \text { Cr } & 50-75 & 35.7 & 29.9 & 28.9 & 5.5 & \text { sandy loam }\end{array}$

R $75+$

Pantohypereutric Leptic Regosols (Pantoarenic, Ochric)/ NEOSSOLO REGOLÍTICO Eutrófico léptico

$\begin{array}{lllllll}\text { A } & 0-5 & 59.5 & 19.6 & 14.0 & 6.9 & \text { loamy sand } \\ \text { AC1 } & 5-15 & 63.9 & 19.1 & 12.6 & 4.4 & \text { loamy sand }\end{array}$




$\begin{array}{lllllll}\text { AC2 } & 15-30 & 65.3 & 15.3 & 15.1 & 4.3 & \text { loamy sand } \\ \text { C } & 30-60 & 59.5 & 15.5 & 15.1 & 9.9 & \text { sandy loam }\end{array}$

R $60+$

Leptic Luvisols (Pantoloamic, Pantohypereutric, Ochric)/ LUVISSOLO CRÔMICO Órtico léptico

$\begin{array}{lllllll}\text { A } & 0-5 & 42.7 & 25.8 & 17.2 & 14.3 & \text { sandy loam } \\ \text { Bt } & 5-25 & 29.3 & 11.7 & 19.1 & 39.9 & \text { clay loam } \\ \text { C } & 25-55 & 43.4 & 20.0 & 13.3 & 23.4 & \text { sandy clay loam } \\ \text { R } & 55+ & & & & & \\ & & & & 17.9- & & \\ \text { All (median-CV) } & & 33.3-39 & 19.3-26 & 34 & 18.0-70\end{array}$

Moist forest soils*

Xanthic Ferralsols (Loamic, Pantohyperdystric, Ochric)/ LATOSSOLO AMARELO Distrófico psamítico

$\begin{array}{lllllll}\text { A1 } & 0-15 & 11.0 & 78.0 & 2.0 & 9.0 & \text { loamy sand } \\ \text { A2 } & 15-30 & 11.0 & 77.0 & 2.0 & 10.0 & \text { loamy sand } \\ \text { Bw1 } & 30-90 & 13.0 & 68.0 & 3.0 & 16.0 & \text { sandy loam } \\ \text { Bw2 } & 90-145 & 13.0 & 68.0 & 2.0 & 17.0 & \text { sandy loam } \\ \text { Bw3 } & 145-175+ & 14.0 & 66.0 & 2.0 & 18.0 & \text { sandy loam }\end{array}$

Xanthic Pisoplinthic Ferralsols (Loamic, Orthodystric, Ferric, Ochric)/ LATOSSOLO AMARELO Distrófico plintossólico

\begin{tabular}{|c|c|c|c|c|c|c|}
\hline A1 & $0-20$ & 20.0 & 60.0 & 6.0 & 14.0 & sandy loam \\
\hline A2 & $20-40$ & 18.0 & 60.0 & 5.0 & 17.0 & sandy loam \\
\hline Bw1 & $40-75$ & 16.0 & 56.0 & 6.0 & 22.0 & sandy clay loam \\
\hline Bw2 & $75-120$ & 18.0 & 54.0 & 6.0 & 22.0 & sandy clay loam \\
\hline Bw3 & $120-165+$ & 17.0 & 45.0 & 8.0 & 30.0 & sandy clay loam \\
\hline
\end{tabular}

Xanthic Pisoplinthic Ferralsols (Loamic, Orthodystric, Ferric, Ochric)/ LATOSSOLO AMARELO Distrófico plintossólico

\begin{tabular}{|c|c|c|c|c|c|c|}
\hline A1 & $0-20$ & 19.0 & 55.0 & 7.0 & 19.0 & sandy loam \\
\hline A2 & $20-40$ & 16.0 & 53.0 & 4.0 & 27.0 & sandy clay loam \\
\hline Bw1 & $40-70$ & 15.0 & 50.0 & 4.0 & 31.0 & sandy clay loam \\
\hline Bw2 & $70-150+$ & 15.0 & 52.0 & 5.0 & 28.0 & sandy clay loam \\
\hline
\end{tabular}

Xanthic Ferric Ferralic Pisoplinthic Acrisols (Loamic, Ochric)/ ARGISSOLO AMARELO Distrófico plintossólico

$\begin{array}{lllllll}\text { A1 } & 0-20 & 19.0 & 68.0 & 4.0 & 9.0 & \text { loamy sand } \\ \text { A2 } & 20-40 & 20.0 & 60.0 & 3.0 & 17.0 & \text { sandy loam } \\ \text { Bw1 } & 40-65 & 19.0 & 53.0 & 4.0 & 24.0 & \text { sandy clay loam } \\ \text { Bw2 } & 65-140+ & 18.0 & 53.0 & 4.0 & 25.0 & \text { sandy clay loam } \\ \text { All (median-CV) } & & 16.5-18 & 58-19 & 4-44 & 18.5-40 & \end{array}$

* (EMBRAPA, 2017).

The reactivity of soils in the semideciduous and dry forest is different. The first one is between extremely and strongly acid, dystrophic, and hyperdystrophic, having aluminum saturation (m) dominantly above $50 \%$ (Table 4). The soils in the dry forest vary from strongly acid to slightly alkaline, hypereutrophic, and $\mathrm{Al}^{3+}$ is virtually absent. $\mathrm{KCl} \mathrm{pH}$ in semideciduous and dry forests soils is equal or below 5.0, and delta $\mathrm{pH}$ is negative in all horizons, indicating the dominance of negative charges. $\mathrm{Ca}^{2+}>\mathrm{Mg}^{2+}>\mathrm{K}^{+}>\mathrm{Na}^{+}$is the base dominance in the exchange complex. In general, BS, ECEC, CEC,

$\begin{array}{lllll}\text { Caminhos de Geografia } \quad \text { Uberlândia-MG } & \text { v. 22, n. } 81 & \text { jun./2021 } & \text { p. 308-328 Página } 318\end{array}$


$\mathrm{PM}, \mathrm{C}$, and $\mathrm{N}$ content in semideciduous forest soils decreases with the increase of depth, suggesting that chemical soil properties are directly correlated to the input of organic residues. In opposite, the increase of natural fertility of dry forest soils with an increase of depth suggests that these soils are directly related to the parent material.

The $\mathrm{C}$ content in the upper horizon of semideciduous forest soils ranged between 0.70 and $2.92 \%$. In the $20-50 \mathrm{~cm}$ depth, the median $\mathrm{C}$ concentration is $1.39 \%$, and the coefficient of variation is $46 \%$. The mean $\mathrm{C}$ content in dry forest soils is slightly lower than in semideciduous forest soils. The $\mathrm{C} / \mathrm{N}$ ratio decreases with the increase of depth. In the uppermost horizons, $\mathrm{C} / \mathrm{N}$ ratio ranged between 10 and 17. In the 10-100-cm depth, the $\mathrm{C} / \mathrm{N}$ ratio values ranged between 2 and 10 (Table 4). The soil profile presented particle and bulk densities values close to the density of quartz (Table 5). The available water and wilting point represent, respectively, 3 and $2 \%$ of total porosity in semideciduous and dry forest soils.

\section{DISCUSSION}

Although semideciduous and dry forest soils are derived from similar parent material, the morphology, texture, and chemical properties suggest that the semideciduous forest soils exhibit common traits with soils in moist and dry forests. The absence of weatherable minerals in the coarse fraction, acidic $\mathrm{pH}$, low $\mathrm{CEC}$, and low base saturation reflects a deeply weathered scenario under moist and tropical climate (Marques et al., 2004; de Souza et al., 2015, 2018), as well as in moist forests (Table 4). On the other hand, coarse sand dominance and low grade structure indicate similarity to incipient soils developed in the Brazilian dry forests (PINHEIRO; COSTA; ARAÚJO, 2013; ARAÚJO FILHO et al., 2017).

The high organic carbon content in the semideciduous forest soils is attributed to the high $\mathrm{C} / \mathrm{N}$ ratio. $\mathrm{A}$ decrease in $\mathrm{C} / \mathrm{N}$ ratio, simultaneous to the proportion of stabilized organic carbon compounds, has been shown in several studies (GIONGO et al., 2011; GRÜNEBERG; ZICHE; WELLBROCK, 2014; PIRES et al., 2017). A strong positive correlation between $\mathrm{C} / \mathrm{N}$ and $\mathrm{C}$ indicates that the organic carbon is stocked in less favorable microbial activity conditions (GRAND and LAVKULICH, 2012). A decrease of potential acidity, ECEC, and CEC with an increase of depth indicates that the soil's fertility from semideciduous forests is not associated with parent material, possibly related to organic carbon. This result indicates that organic carbon influences geochemical processes in semideciduous forests and moist forests (CARTER, 2000; SOUZA et al., 2015).

Low clay content is attributed to gleization, ferrolysis, and elutriation. Elutriation is a common soil process in semiarid areas of selective transport of lighter particles in the surface horizon by rainwater. The low to moderate grades in this study area suggest high erosivity of rains in these soils, so the clay can be preferentially eroded to lower altitudes. Kaolinite and Fe (hydr)oxides are the main components of the clay fraction in acidic Brazilian soils (FONTES and WEED, 1991). However, poor drainage and waterlogged conditions in Stagnosols are associated with reductive dissolution of $\mathrm{Fe}$ (hydr)oxides. Values of $\mathrm{P}_{\mathrm{REM}}$ above $20 \mathrm{mg} \mathrm{L}^{-1}$ indicate a low $\mathrm{P}$ adsorption capacity and it suggests a virtual absence of $\mathrm{Fe}$ oxides in these soils. Besides, seasonally alternating cycles of reduction and oxidation lead to clay destruction by ferrolysis and CEC reduction (BRINKMAN, 1970; FERREIRA et al., 2016). During the anaerobic phase, iron is reduced by microorganisms with the oxidation of organic matter and the formation of hydroxyl ions. The $\mathrm{Fe}^{2+}$ displaces exchangeable cations, which are leached. During the following aerobic phase, $\mathrm{Fe}(\mathrm{OH})_{3}$ and $\mathrm{H}^{+}$are produced by oxidation of $\mathrm{Fe}^{2+}$. Consequently, the $\mathrm{H}^{+}$ displaces the exchangeable ferrous iron, and it corrodes the octahedral layers of the clay minerals at their edges. Simultaneously, there is an equivalent diffusion of hydrogen against bases released from the octahedral lattice edges. With continued fluctuation between anaerobic and aerobic phases, the seasonally eutrophic soil develops to a grey, unstable sandy soil with low clay content and very low cation exchange capacity. Widespread redoximorphic colors are attributed to stagnation of rainwater in deep horizons due to low permeable layers on subsurface. This process is different from the rise of the water table and it changes the soil morphology. Furthermore, we suggest the inclusion of the suborder 'Estágnicos' for Gleissolos in the Brazilian Soil Classification System, once soils with stagnic properties have a larger spatial distribution than expected.

Caminhos de Geografia

Uberlândia-MG

\begin{abstract}
v. 22, n. 81
\end{abstract}
jun./2021

p. $308-328$

Página 319 
Table 4 - Borborema province: Chemical soil properties of soils in the study area and comparison data.

\begin{tabular}{|c|c|c|c|c|c|c|c|c|c|c|c|c|c|c|c|c|c|c|c|}
\hline $\begin{array}{l}\text { Horiz } \\
\text { on }\end{array}$ & $\begin{array}{l}\text { Depth } \\
\text { cm }\end{array}$ & $\begin{array}{l}\mathrm{H}_{2} \mathrm{O} \\
\mathrm{pH}\end{array}$ & $\begin{array}{l}\mathrm{KCl} \\
\mathrm{pH}\end{array}$ & $\mathbf{P}_{\mathbf{M}}$ & $\begin{array}{c}\mathrm{K}^{+} \\
--\mathrm{mg} \mathrm{k}\end{array}$ & $\mathrm{Na}^{+}$ & $\mathrm{Ca}^{2+}$ & $\mathrm{Mg}^{2+}$ & $\mathbf{A l}^{3+}$ & $\mathrm{H}+\mathrm{Al}$ & $g^{-1}----$ & ECEC & CEC & V & ---- & 6 ------- & $\mathbf{N}$ & $\mathrm{C} / \mathrm{N}$ & $\begin{array}{l}P_{\text {REM }} \\
\mathrm{mg}^{-1} \\
\mathrm{~L}^{-1}\end{array}$ \\
\hline \multicolumn{20}{|c|}{ Semideciduous forest soils } \\
\hline \multicolumn{20}{|c|}{ Leptic Umbrisol (Endoclayic, Hyperdystric)/ NEOSSOLO REGOLÍTICO Húmico léptico } \\
\hline$A_{1}$ & $0-20$ & 4.4 & 3.67 & 3.3 & 90 & 7.8 & 1.82 & 1.00 & 1.8 & 12.7 & 3.08 & 4.83 & 15.78 & 20 & 36 & 2.92 & 0.224 & 13 & 26.6 \\
\hline $\mathrm{A}_{2}$ & $20-30$ & 4.38 & 3.69 & 1.8 & 47 & 4.9 & 0.65 & 0.80 & 2.2 & 11.6 & 1.59 & 3.83 & 13.19 & 12 & 59 & 2.55 & 0.153 & 17 & 24.2 \\
\hline$A C$ & $30-50$ & 4.69 & 3.79 & $<1$ & 71 & 9.8 & 0.29 & 1.49 & 1.9 & 8.7 & 2.00 & 3.85 & 10.7 & 19 & 48 & 1.39 & 0.102 & 14 & 22.2 \\
\hline $\mathrm{Cr}$ & $50-55$ & 4.75 & 3.85 & $<1$ & 76 & 11.8 & 0.22 & 1.51 & 1.5 & 5.6 & 1.98 & 3.44 & 7.58 & 26 & 42 & 0.70 & 0.067 & 10 & 22.7 \\
\hline $\mathrm{R}$ & $55+$ & & & & & & & & & & & & & & & & & & \\
\hline \multicolumn{20}{|c|}{ Haplic Umbrisol (Pantoloamic, Pantohyperdystric)/ NEOSSOLO REGOLÍTICO Húmico típico } \\
\hline$A_{1}$ & $0-40$ & 4.35 & 3.69 & 2.4 & 76 & $<1$ & 0.85 & 0.46 & 2.7 & 12 & 1.51 & 4.23 & 13.51 & 11 & 64 & 2.39 & 0.142 & 17 & 24.7 \\
\hline$A_{2}$ & $40-62$ & 4.42 & 3.70 & 1.2 & 20 & 3.9 & 0.39 & 0.38 & 3.1 & 11.4 & 0.84 & 3.95 & 12.24 & 7 & 79 & 1.39 & 0.085 & 16 & 21.8 \\
\hline $\mathrm{A}_{3}$ & $62-100$ & 4.73 & 3.70 & 1.0 & 16 & 12.8 & 0.51 & 0.54 & 2.8 & 9.2 & 1.15 & 3.97 & 10.35 & 11 & 71 & 0.93 & 0.057 & 16 & 23.1 \\
\hline$A C$ & $100-140$ & 5.15 & 3.75 & 1.0 & 24 & 37.5 & 0.49 & 0.95 & 2.2 & 6.9 & 1.66 & 3.90 & 8.56 & 19 & 57 & 0.54 & 0.042 & 13 & 27 \\
\hline $\mathrm{R}$ & $140+$ & & & & & & & & & & & & & & & & & & \\
\hline \multicolumn{20}{|c|}{ Pantoorthodystric Stagnosols (Amphiclayic, Katomanganiferric, Humic, Inclinic)/ GLEISSOLO HÁPLICO Tb Distrófico argissólico } \\
\hline A & $0-25$ & 4.6 & 3.82 & 3.9 & 73 & $<1$ & 1.3 & 0.76 & 0.7 & 8 & 2.25 & 2.93 & 10.25 & 22 & 23 & 2.55 & 0.165 & 16 & 39 \\
\hline$A B$ & $25-60$ & 4.44 & 3.87 & 1.2 & 27 & $<1$ & 0.27 & 0.16 & 1.7 & 6.9 & 0.50 & 2.15 & 7.4 & 7 & 77 & 1.00 & 0.062 & 16 & 28.3 \\
\hline Btg & $\begin{array}{l}70-90(60- \\
100)\end{array}$ & 4.69 & 3.88 & $<1$ & 32 & $<1$ & 0.28 & 0.44 & 1.9 & 6.4 & 0.81 & 2.66 & 7.21 & 11 & 70 & 0.62 & 0.050 & 12 & 24.2 \\
\hline Cgr & $90-150+$ & 4.85 & 3.94 & $<1$ & 32 & 4.9 & 0.21 & 1.00 & 1.5 & 5.1 & 1.31 & 2.77 & 6.41 & 20 & 53 & 0.62 & 0.043 & 14 & 23.1 \\
\hline \multicolumn{20}{|c|}{ Pantoorthodystric Anoumbric Stagnosols (Pantoclayic, Humic, Inclinic)/ GLEISSOLO MELÂNICO Tb Distrófico típico } \\
\hline $\mathrm{Ag}$ & $0-70$ & 4.92 & 3.79 & 1.6 & 32 & 9.8 & 0.5 & 0.52 & 1.4 & 5.5 & 1.14 & 2.50 & 6.64 & 17 & 54 & 0.85 & 0.053 & 16 & 33.2 \\
\hline CAg & $70-122$ & 5.3 & 4.02 & $<1$ & 18 & 7.8 & 0.32 & 0.27 & 0.4 & 1.9 & 0.67 & 1.06 & 2.57 & 26 & 37 & 0.08 & 0.009 & 9 & 52.6 \\
\hline $\mathrm{Cg}$ & $122-160+$ & 5.23 & 4.00 & $<1$ & 20 & 10.8 & 0.29 & 0.33 & 0.6 & 1.8 & 0.72 & 1.30 & 2.52 & 29 & 45 & $<0.01$ & 0.005 & 2 & 51.6 \\
\hline \multicolumn{20}{|c|}{ Leptic Umbrisol (Endoclayic, Pantodystric)/ NEOSSOLO REGOLÍTICO Húmico léptico } \\
\hline$A_{1}$ & $0-27$ & 4.94 & 3.92 & 3.7 & 154 & 15.7 & 1.99 & 1.12 & 0.8 & 6.9 & 3.57 & 4.35 & 10.47 & 34 & 18 & 2.39 & 0.187 & 13 & 38.7 \\
\hline $\mathrm{A}_{2}$ & $27-47$ & 4.85 & 3.80 & 1.6 & 114 & 20.7 & 0.61 & 1.05 & 1.9 & 6.9 & 2.04 & 3.89 & 8.94 & 23 & 48 & 1.39 & 0.093 & 15 & 27.6 \\
\hline $\mathrm{Cr}$ & $47-67$ & 5.00 & 3.75 & $<1$ & 78 & 35.5 & 0.39 & 1.52 & 2.5 & 5.5 & 2.26 & 4.79 & 7.76 & 29 & 53 & 0.31 & 0.039 & 8 & 27.8 \\
\hline क्षा & 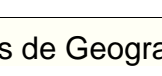 & & & & & - & & & $\cdot 2<, 11$ & & & 年 & & & 00 & & & 320 & \\
\hline
\end{tabular}


R $67+$

Pantoorthodystric Anoumbric Stagnosols (Pantoclayic, Katomanganiferric, Humic, Inclinic)/GLEISSOLO MELÂNICO Tb Distrófico típico

\begin{tabular}{|c|c|c|c|c|c|c|c|c|c|c|c|c|c|c|c|c|c|c|c|}
\hline A & $0-58$ & 4.56 & 3.66 & 1.8 & 101 & 1.9 & 0.49 & 0.22 & 1.7 & 6.6 & 0.98 & 2.63 & 7.58 & 13 & 63 & 0.70 & 0.071 & 10 & 34.9 \\
\hline $\mathrm{CA}$ & $58-78$ & 4.57 & 3.75 & $<1$ & 27 & $<1$ & 0.27 & 0.08 & 1.3 & 3.9 & 0.42 & 1.69 & 4.32 & 10 & 75 & 0.23 & 0.021 & 11 & 42.8 \\
\hline $\mathrm{Cg}$ & $78-150+$ & 5.16 & 3.67 & $<1$ & 32 & 1.9 & 0.36 & 0.22 & 0.7 & 2.1 & 0.67 & 1.35 & 2.77 & 24 & 50 & 0.08 & 0.008 & 10 & 51.2 \\
\hline \multicolumn{20}{|c|}{ Leptic Umbrisol (Pantoloamic, Hyperdystric)/ NEOSSOLO REGOLÍTICO Distrófico léptico } \\
\hline A & $0-45$ & 4.62 & 3.70 & 4.1 & 61 & 2.9 & 1.32 & 0.64 & 0.8 & 6.6 & 2.13 & 2.91 & 8.73 & 24 & 27 & 1.16 & 0.085 & 14 & 45.5 \\
\hline AC & $45-100$ & 4.79 & 3.89 & $<1$ & 39 & 3.9 & 0.28 & 0.26 & 1.0 & 3.5 & 0.66 & 1.63 & 4.16 & 16 & 60 & 0.16 & 0.018 & 9 & 42.9 \\
\hline $\mathrm{R}$ & $100+$ & & & & & & & & & & & & & & & & & & \\
\hline \multicolumn{2}{|c|}{ All (median-CV) } & $\begin{array}{l}4.73- \\
6\end{array}$ & $\begin{array}{l}3.79- \\
3\end{array}$ & $\begin{array}{l}1- \\
129\end{array}$ & $39-93$ & $\begin{array}{l}4.9- \\
209\end{array}$ & $\begin{array}{l}0.39- \\
130\end{array}$ & $\begin{array}{l}0.54- \\
83\end{array}$ & $\begin{array}{l}1.7- \\
47\end{array}$ & $\begin{array}{l}6.6- \\
47\end{array}$ & $\begin{array}{l}1.31- \\
64\end{array}$ & $\begin{array}{l}2.93- \\
39\end{array}$ & $\begin{array}{l}7.76- \\
46\end{array}$ & $\begin{array}{l}19.4- \\
39\end{array}$ & $\begin{array}{l}52.8- \\
31\end{array}$ & $\begin{array}{l}0.85- \\
83\end{array}$ & $\begin{array}{l}0.077- \\
78\end{array}$ & $\begin{array}{l}13- \\
29\end{array}$ & $\begin{array}{l}27.8- \\
38\end{array}$ \\
\hline
\end{tabular}

Dry forest soils

Leptic Luvisols (Pantoloamic, Pantohypereutric, Ochric)/ LUVISSOLO CRÔMICO Órtico léptico

\begin{tabular}{|c|c|c|c|c|c|c|c|c|c|c|c|c|c|c|c|c|c|c|c|}
\hline A & $0-10$ & 7.26 & 5.07 & 1.5 & 34 & 97.9 & 16.88 & 8.68 & 0.0 & 0.8 & 26.07 & 26.07 & 26.87 & 97 & 0 & 0.31 & 0.004 & 81 & 40.9 \\
\hline $\mathrm{Bt}$ & $10-30$ & 7.57 & 5.47 & 13.8 & 19 & 149.3 & 16.41 & 7.32 & 0.0 & 0.6 & 24.43 & 24.43 & 25.03 & 98 & 0 & 0.31 & 0.003 & 123 & 48 \\
\hline CR & $30-50$ & 8.12 & 5.31 & $\begin{array}{l}107 . \\
7\end{array}$ & 11 & 239.2 & 21.38 & 8.87 & 0.0 & 0.3 & 31.32 & 31.32 & 31.62 & 99 & 0 & 0.08 & 0.008 & 9 & 51.2 \\
\hline
\end{tabular}

R 50+

Rhodic Luvisols (Pantoloamic, Pantohypereutric, Ochric)/ LUVISSOLO CRÔMICO Órtico típico

$\begin{array}{llllllllllllllllllll}\mathrm{A} & 0-3(2-5) & 7.19 & 6.33 & 2 & 329 & 19.6 & 14.25 & 4.75 & 0.0 & 1.1 & 19.93 & 19.93 & 21.03 & 95 & 0 & 1.57 & 0.089 & 18 & 36.5 \\ \mathrm{Bt}_{1} & 3-25 & 6.64 & 4.85 & 7.6 & 85 & 49.4 & 22.57 & 9.25 & 0.0 & 1.5 & 32.25 & 32.25 & 33.75 & 96 & 0 & 0.39 & 0.029 & 13 & 32.8 \\ \mathrm{Bt}_{2} & 25-50 & 6.90 & 4.27 & 15.7 & 70 & 85.1 & 27.12 & 9.86 & 0.0 & 1.5 & 37.53 & 37.53 & 39.03 & 96 & 0 & 0.20 & 0.012 & 17 & 35.1 \\ \mathrm{Cr} & 50-75 & 6.90 & 4.02 & 52.6 & 52 & 71.2 & 22.67 & 7.23 & 0.0 & 1.0 & 30.34 & 30.34 & 31.34 & 97 & 0 & 0.20 & 0.012 & 17 & 43.9\end{array}$

R 75+

Pantohypereutric Leptic Regosols (Pantoarenic, Ochric)/ NEOSSOLO REGOLÍTICO Eutrófico léptico

\begin{tabular}{|c|c|c|c|c|c|c|c|c|c|c|c|c|c|c|c|c|c|c|c|}
\hline A & $0-5$ & 7.16 & 6.53 & 10.7 & 115 & 7.7 & 8.07 & 1.69 & 0.3 & 0.6 & 10.09 & 10.38 & 10.69 & 94 & 3 & 0.20 & 0.02 & 10 & 44.9 \\
\hline$A C_{1}$ & $5-15$ & 6.71 & 5.58 & 3.2 & 74 & 3.7 & 3.42 & 0.96 & 0.0 & 1.0 & 4.59 & 4.59 & 5.59 & 82 & 0 & 0.47 & 0.053 & 9 & 45.7 \\
\hline $\mathrm{AC}_{2}$ & $15-30$ & 6.70 & 5.30 & 2.7 & 48 & 7.7 & 3.00 & 1.40 & 0.0 & 0.8 & 4.56 & 4.56 & 5.36 & 85 & 0 & 0.24 & 0.041 & 6 & 47.4 \\
\hline C & $30-60$ & 6.10 & 4.41 & 2.6 & 38 & 29.5 & 2.73 & 1.67 & 000 & 1.3 & 4.63 & 4.63 & 5.93 & 78 & 0 & 0.16 & 0.003 & 53 & 42.7 \\
\hline
\end{tabular}

$\begin{array}{ll}\mathrm{C} & 30-60 \\ \mathrm{R} & 60+\end{array}$

Leptic Luvisols (Pantoloamic, Pantohypereutric, Ochric)/ LUVISSOLO CRÔMICO Órtico léptico 


\begin{tabular}{|c|c|c|c|c|c|c|c|c|c|c|c|c|c|c|c|c|c|c|c|}
\hline A & $0-5$ & 6.30 & 5.15 & 6.0 & 165 & 13.6 & 6.58 & 2.82 & 0.0 & 1.6 & 9.88 & 9.88 & 11.48 & 86 & 0 & 0.79 & 0.069 & 11 & 41.4 \\
\hline $\mathrm{Bt}$ & $5-25$ & 5.74 & 3.48 & 2.3 & 52 & 505.0 & 21.80 & $\begin{array}{l}17.5 \\
2\end{array}$ & 0.5 & 2.3 & 41.65 & 42.14 & 43.95 & 95 & 1 & 0.31 & 0.02 & 16 & 37.0 \\
\hline C & $25-55$ & 5.22 & 3.86 & 3.1 & 56 & 69.2 & 7.60 & 5.15 & 0.2 & 2.7 & 13.19 & 13.39 & 15.89 & 83 & 2 & 0.31 & 0.02 & 16 & 34.7 \\
\hline $\mathrm{R}$ & $55+$ & & & & & & & & & & & & & & & & & & \\
\hline \multicolumn{2}{|c|}{ All (median-CV) } & $\begin{array}{l}6.8- \\
11 \\
\end{array}$ & $\begin{array}{l}5.11- \\
18 \\
\end{array}$ & $\begin{array}{l}6.8- \\
179 \\
\end{array}$ & $54-99$ & $\begin{array}{l}59.3- \\
140 \\
\end{array}$ & $\begin{array}{l}15.33- \\
61 \\
\end{array}$ & $\begin{array}{l}6.19- \\
74 \\
\end{array}$ & $\begin{array}{l}<1- \\
215\end{array}$ & $\begin{array}{l}1.1- \\
54\end{array}$ & $\begin{array}{l}22.18- \\
62 \\
\end{array}$ & $\begin{array}{l}22.18- \\
62 \\
\end{array}$ & $\begin{array}{l}23.03- \\
59\end{array}$ & $95-7$ & $\begin{array}{l}<1- \\
218\end{array}$ & $\begin{array}{l}0.31- \\
112 \\
\end{array}$ & $\begin{array}{l}0.02- \\
134 \\
\end{array}$ & $\begin{array}{l}16- \\
120\end{array}$ & $\begin{array}{l}42.1- \\
14 \\
\end{array}$ \\
\hline \multicolumn{20}{|c|}{ Moist forests soils* } \\
\hline \multicolumn{20}{|c|}{ Xanthic Ferralsols (Loamic, Pantohyperdystric, Ochric)/ LATOSSOLO AMARELO Distrófico psamítico } \\
\hline$A_{1}$ & $0-15$ & 5.2 & 4.4 & 1.0 & 1.17 & 0.23 & $<1$ & $<1$ & 0.2 & 0.6 & 2.4 & 0.8 & 3.2 & 19 & 22 & 5.3 & 0.5 & 11 & - \\
\hline$A_{2}$ & $15-300$ & 5.2 & 4.6 & 1.0 & 1.17 & 0.23 & $<1$ & $<1$ & 0.2 & 0.4 & 2 & 0.6 & 2.6 & 15 & 29 & 4 & 0.5 & 8 & - \\
\hline$B w_{1}$ & $30-90$ & 5.2 & 4.6 & 1.0 & 0.78 & 0.23 & $<1$ & $<1$ & 0.3 & 0.2 & 2.1 & 0.5 & 2.6 & 8 & 60 & 4.8 & 0.5 & 10 & - \\
\hline $\mathrm{Bw}_{2}$ & $90-145$ & 5.1 & 4.6 & 1.0 & 1.17 & 0.46 & $<1$ & $<1$ & 0.2 & 0.3 & 1.8 & 0.5 & 2.3 & 13 & 40 & 3.4 & 0.4 & 9 & - \\
\hline $\mathrm{Bw}_{3}$ & $145-175+$ & 5.2 & 4.5 & 1.0 & 1.17 & 0.46 & $<1$ & $<1$ & 0.3 & 0.3 & 1.5 & 0.6 & 2.1 & 14 & 50 & 1.8 & 0.3 & 6 & - \\
\hline \multicolumn{20}{|c|}{ Xanthic Pisoplinthic Ferralsols (Loamic, Orthodystric, Ferric, Ochric)/ LATOSSOLO AMARELO Distrófico plintossólico } \\
\hline$A_{1}$ & $0-20$ & 6.8 & 6.2 & 4.0 & 3.9 & 1.15 & 3.1 & 1.2 & 0 & 4.5 & 0.4 & 4.5 & 4.9 & 92 & 0 & 10.5 & 1.1 & 10 & - \\
\hline $\mathrm{A}_{2}$ & $20-40$ & 5.3 & 3.9 & 1.0 & 5.07 & 0.92 & $<1$ & $<1$ & 0.4 & 0.9 & 1.3 & 1.3 & 2.6 & 41 & 31 & 4.1 & 0.4 & 10 & - \\
\hline$B w_{1}$ & $40-75$ & 5.1 & 4.0 & 1.0 & 10.92 & 0.92 & $<1$ & $<1$ & 0.3 & 1.2 & 1.8 & 1.5 & 3.3 & 36 & 20 & 3.4 & 0.4 & 9 & - \\
\hline $\mathrm{Bw}_{2}$ & $75-120$ & 5.0 & 3.9 & 1.0 & 2.73 & 1.15 & $<1$ & $<1$ & 0.3 & 1 & 1.1 & 1.3 & 2.4 & 42 & 21 & 3.3 & 0.3 & 11 & - \\
\hline $\mathrm{Bw}_{3}$ & $120-165+$ & 5.0 & 3.9 & $>1$ & 1.56 & 2.76 & 1.1 & 0.3 & 0.4 & 1.6 & 1.5 & 2 & 3.5 & 46 & 20 & 3.4 & 0.3 & 11 & - \\
\hline \multicolumn{20}{|c|}{ Xanthic Pisoplinthic Ferralsols (Loamic, Orthodystric, Ferric, Ochric)/LATOSSOLO AMARELO Distrófico plintossólico } \\
\hline A1 & $0-20$ & 5.0 & 3.8 & 2.0 & 1.56 & 1.15 & 1.9 & 0.5 & 0.5 & 2.5 & 7.3 & 3 & 10.3 & 24 & 17 & 18.9 & 1.6 & 12 & - \\
\hline $\mathrm{A} 2$ & $20-40$ & 5.1 & 3.9 & 2.0 & 1.56 & 1.15 & 1.9 & 0.5 & 0.7 & 2.5 & 5.6 & 3.2 & 7.7 & 18 & 33 & 18.9 & 1.6 & 12 & - \\
\hline Bw1 & $40-70$ & 5.2 & 3.9 & $>1$ & 2.73 & 0.92 & 1 & $<1$ & 0.6 & 1.1 & 3.5 & 1.7 & 5.2 & 21 & 35 & 7.1 & 0.5 & 14 & - \\
\hline Bw2 & $70-150+$ & 5.1 & 4 & $<1$ & 0.39 & 0.69 & 0.6 & $<1$ & 0.7 & 0.6 & 2.7 & 1.3 & 4 & 15 & 54 & 4.9 & 0.3 & 16 & - \\
\hline \multicolumn{20}{|c|}{ Xanthic Ferric Ferralic Pisoplinthic Acrisols (Loamic, Ochric)/ARGISSOLO AMARELO Distrófico plintossólico } \\
\hline$A_{1}$ & $0-20$ & 5.1 & 4.1 & 1.0 & 0.78 & 0.46 & $<1$ & $<1$ & 0.2 & 0.4 & 1.4 & 0.6 & 2 & 20 & 33 & 2.7 & 0.4 & 7 & - \\
\hline$A_{2}$ & $20-40$ & 5.0 & 4.2 & 1.0 & 0.39 & 0.46 & $<1$ & $<1$ & 0.2 & 0.2 & 1.6 & 0.4 & 2 & 10 & 50 & 2.4 & 0.4 & 6 & - \\
\hline$B w_{1}$ & $40-65$ & 5.1 & 4.4 & 1.0 & 0.39 & 0.46 & $<1$ & $<1$ & 0.2 & 0.3 & 1.8 & 0.5 & 2.3 & 13 & 40 & 2.2 & 0.3 & 7 & - \\
\hline $\mathrm{Bw}_{2}$ & $65-140+$ & 4.9 & 4.3 & 1.0 & 0.39 & 0.46 & $<1$ & $<1$ & 0.2 & 0.3 & 1.7 & 0.5 & 2.2 & 14 & 40 & 2 & 0.3 & 7 & - \\
\hline All $(m$ & dian-CV) & $5.1-8$ & $\begin{array}{l}4.15- \\
13\end{array}$ & $\begin{array}{l}1.0- \\
61\end{array}$ & $\begin{array}{l}1.17- \\
121\end{array}$ & $\begin{array}{l}0.58- \\
75\end{array}$ & $1.5-56$ & $\begin{array}{l}0.5- \\
63\end{array}$ & $\begin{array}{l}0.3- \\
58\end{array}$ & $\begin{array}{l}0.6- \\
106\end{array}$ & $1.8-72$ & $1.1-83$ & $2.6-61$ & $\begin{array}{l}18.5- \\
79\end{array}$ & $\begin{array}{l}33- \\
46\end{array}$ & 3.7-91 & $0.4-75$ & $\begin{array}{l}10- \\
27\end{array}$ & - \\
\hline \multicolumn{20}{|c|}{${ }^{*}$ (EMBRAPA, 2017). } \\
\hline Camint & s de Geogra & & & & Ube & ândia-N & & & $22, n$ & & & un./2021 & & & 308 & & Págir & a 322 & \\
\hline
\end{tabular}


Table 5 - Borborema province: Physical soil properties determined in undisturbed samples.

\begin{tabular}{|c|c|c|c|c|c|c|c|c|c|c|c|}
\hline \multirow[t]{3}{*}{ Horizon } & \multirow[t]{2}{*}{ Depth } & \multirow[t]{2}{*}{ Particles density } & \multirow[t]{2}{*}{ Bulk density } & \multirow[t]{2}{*}{ Total porosity } & \multicolumn{6}{|c|}{ Water retention curve $(\mathrm{kPa})$} & \multirow[t]{2}{*}{ Available water } \\
\hline & & & & & -6 & -10 & -30 & -100 & -300 & -1500 & \\
\hline & $\mathbf{c m}$ & --------- kg/ kg --- & & ------------------. & $--\%$ & ----- & ----- & & & & \\
\hline
\end{tabular}

Leptic Umbrisol (Endoclayic, Hyperdystric)/ NEOSSOLO REGOLÍTICO Húmico léptico

$\begin{array}{llllllllllll}\mathrm{A}_{1} & 0-20 & 2.61 & 1.38 & 29.68 & 25.0 & 19.6 & 16.5 & 13.8 & 10.3 & 7.5 & 12.1 \\ \mathrm{~A}_{2} & 20-30 & 2.71 & 1.56 & 27.97 & 24.3 & 20.9 & 17.5 & 15.5 & 13.4 & 9.0 & 11.9\end{array}$

Haplic Umbrisol (Pantoloamic, Pantohyperdystric)/ NEOSSOLO REGOLÍTICO Húmico típico

\begin{tabular}{|c|c|c|c|c|c|c|c|c|c|c|c|}
\hline$A_{1}$ & $0-40$ & 2.74 & 1.60 & 33.93 & 28.7 & 21.8 & 19.1 & 17.1 & 15.6 & 1.0 & 20.8 \\
\hline$A_{2}$ & $40-62$ & 2.74 & 1.65 & 37.00 & 30.2 & 20.3 & 17.8 & 15.9 & 13.8 & 1.0 & 19.3 \\
\hline
\end{tabular}

Pantoorthodystric Stagnosols (Amphiclayic, Katomanganiferric, Humic, Inclinic)/ GLEISSOLO HÁPLICO Tb Distrófico argissólico

\begin{tabular}{|c|c|c|c|c|c|c|c|c|c|c|c|}
\hline A & $0-25$ & 2.15 & 0.99 & 25.53 & 21.3 & 15.1 & 13.5 & 11.8 & 9.7 & 5.7 & 9.4 \\
\hline Btg & $70-90(60-100)$ & 2.79 & 1.63 & 38.26 & 31.7 & 22.4 & 19.7 & 17.9 & 13.1 & 9.6 & 12.8 \\
\hline
\end{tabular}

Pantoorthodystric Anoumbric Stagnosols (Pantoclayic, Humic, Inclinic)/ GLEISSOLO MELÂNICO Tb Distrófico típico
$\mathrm{Ag}$
$0-70$
2.78
1.71
20.97

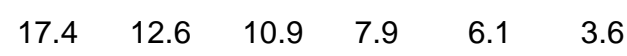
9.0

Leptic Umbrisol (Endoclayic, Pantodystric)/ NEOSSOLO REGOLÍTICO Húmico léptico
$A_{1}$
$0-27$
2.67
1.39
28.55
$24.2 \quad 18.5 \quad 16.2 \quad 14.8 \quad 12.7 \quad 9.8$
8.7

Pantoorthodystric Anoumbric Stagnosols (Pantoclayic, Katomanganiferric, Humic, Inclinic)/GLEISSOLO MELÂNICO Tb Distrófico típico 


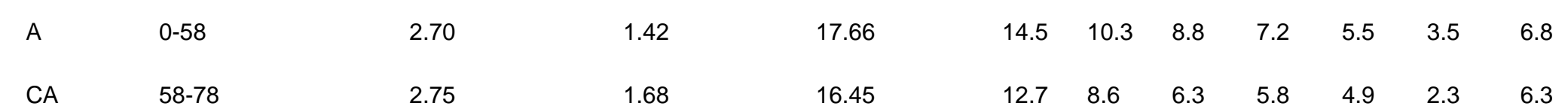

Leptic Umbrisol (Pantoloamic, Hyperdystric)/ NEOSSOLO REGOLÍTICO Distrófico léptico

\begin{tabular}{|c|c|c|c|c|c|c|c|c|c|c|c|}
\hline A & $0-45$ & 2.65 & 1.35 & 18.11 & 14.0 & 7.9 & 6.7 & 5.8 & 4.7 & 2.7 & 5.2 \\
\hline$A C$ & $45-100$ & 2.73 & 1.6 & 22.17 & 18.4 & 13.8 & 11.6 & 8.8 & 6.1 & 3.3 & 10.5 \\
\hline
\end{tabular}

Dry forest soils

Leptic Luvisols (Pantoloamic, Pantohypereutric, Ochric)/ LUVISSOLO CRÔMICO Órtico léptico

$\begin{array}{llllllllllll}\text { A } & 0-10 & 2.71 & 1.42 & 35.97 & 31.8 & 25.8 & 23.9 & 20.8 & 18.1 & 13.5 & 12.3 \\ \mathrm{Bt} & 10-30 & 2.72 & 1.66 & 30.38 & 27.6 & 24.4 & 22.3 & 19.7 & 16.1 & 12.6 & 11.8\end{array}$

Rhodic Luvisols (Pantoloamic, Pantohypereutric, Ochric)/ LUVISSOLO CRÔMICO Órtico típico

$\begin{array}{llllllllllll}\text { A } & 0-3(2-5) & 2.68 & 1.27 & 52.61 & 40.5 & 17.5 & 15.7 & 13.7 & 11.1 & 8.3 & 9.2 \\ \mathrm{Bt} 1 & 3-25 & 2.65 & 1.21 & 54.33 & 36.9 & 19.0 & 16.6 & 14.1 & 12.7 & 8.7 & 10.3\end{array}$

Pantohypereutric Leptic Regosols (Pantoarenic, Ochric)/ NEOSSOLO REGOLÍTICO Eutrófico léptico

\begin{tabular}{|c|c|c|c|c|c|c|c|c|c|c|c|}
\hline A & $0-5$ & 2.90 & 1.53 & 47.24 & 27.2 & 6.5 & 5.2 & 4.9 & 3.7 & 2.2 & 4.3 \\
\hline$A C_{1}$ & $5-15$ & 2.87 & 1.46 & 49.12 & 24.3 & 7.1 & 5.6 & 4.9 & 4.2 & 2.5 & 4.6 \\
\hline
\end{tabular}

Leptic Luvisols (Pantoloamic, Pantohypereutric, Ochric)/ LUVISSOLO CRÔMICO Órtico léptico

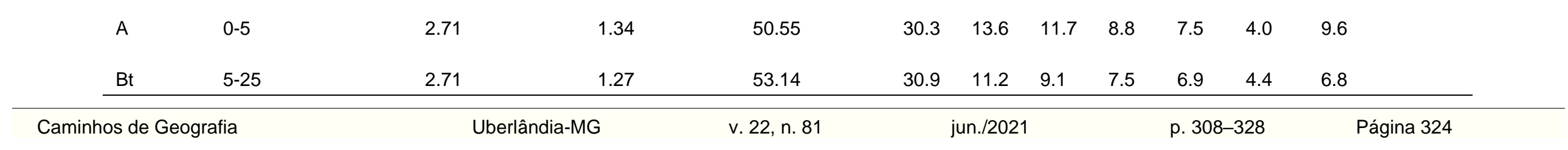


The higher amount of available water and lower wilting point in semideciduous forest compared to dry forests could be used to explain the differences between these two ecosystems. Once differences in precipitation between these sites are low, the higher the available amount of water, the larger water volume could be consumed during the year. Simultaneously, a lower wilting point indicates that soil moisture can achieve a lower level without restricting water absorption by plants. The lower water availability in semiarid regions limits the nutrient absorption by plants, as $N$ and P (SILVEIRA et al., 2006; MENEZES et al., 2012). Drought resistance species are less affected and persist while sensitive species have grown and reproduces compromised (BARROS et al., 2018; TERRA et al., 2018). Higher $\mathrm{C}, \mathrm{N}$, and $\mathrm{P}_{\mathrm{M}}$ and contents guarantee the nutrition of several organisms and promote change in the composition of the vegetation community. Consequently, higher diversity and richness favor higher primary productivity, partially incorporated into the soil as organic residues. These organic residues became soil organic matter and contribute to soil fertility (MINICK; FISK; GROFFMAN, 2017; NOVOTNY et al., 2007) and water retention capacity (GAISER; GRAEF; CORDEIRO, 2000; VALARINI et al., 2003). This biogeochemical cycling of nutrients improves efficiency in a restrictive region. Previous studies indicated that where availability of nutrients is restricted to primary productivity a that higher efficiency in the use of natural resources decreases competition for nutrients and favors the occurrence of a higher number of species (PELLEGRINI, 2016; PEÑA-CLAROS et al., 2012; TILMAN et al., 2001).

\section{CONCLUSIONS}

Our results emphasize that the semideciduous forest has characteristics found in humid and dry forests, reinforcing the results of other authors that these environments are essential to transition areas. The soils of dry and semideciduous forests are derived from similar parent materials, have similar textures with a coarse sand domain. However, soils in semideciduous forests have lower CEC and base saturation values than those in dry forest soils. This difference is attributed to the orographic rains captured by the eastern slope in the province of Borborema and, consequently, more significant weathering and leaching of bases, making the soils of semideciduous forest resemble those of humid forest. The higher amount of available water and the lower wilt point in the semideciduous forest than dry forests can be highlighted as one of the main diagnostic characteristics responsible for separating these two ecosystems. With greater water availability throughout the year, semideciduous forests can house species adapted to water stress as less adapted species, contributing to specific vegetation composition.

We emphasize that the study of semideciduous forest soils is essential to clarify doubts about the correct characterization and classification of these environments, in addition to serving as a basis for future management plans and study of forest restoration, since detailing of soil characteristics can determine which species best adapt to the environment of interest.

\section{REFERENCES}

ALLIÉ, E., PÉLISSIER, R., ENGEL, J., PETRONELLI, P., FREYCON, V., DEBLAUWE, V., SOUCÉMARIANADIN, L., WEIGEL, J., BARALOTO, C., 2015. Pervasive local-scale tree-soil habitat association in a tropical forest community. PLoS One, v. 10, p. 1-16, 2015.

https://doi.org/10.1371/journal.pone.0141488

ALVAREZ V., V.H., NOVAIS, R.F., DIAS, L.E., OLIVEIRA, J.A. Determinação e uso do fósforo remanescente. Boletim Informativo da Sociedade Brasileira de Ciência do Solo, v. 25, p. 27-32, 2000.

ARAÚJO FILHO, J.C., RIBEIRO, M.R., BURGOS, N., MARQUES, F.A. SOLOS DA CAATINGA. In: CURI, N., KER, J.C., NOVAIS, R.F., VIDAL-TORRADO, P., SCHAEFER, C.E.G.R. (Eds.), Pedologia - Solos Dos Biomas Brasileiros. Viçosa : Sociedade Brasileira de Ciência do Solo, 2017, p. 227-260.

ARRUDA, D.M., FERNANDES-FILHO, E.I., SOLAR, R.R.C., SCHAEFER, C.E.G.R., Combining climatic and soil properties better predicts covers of Brazilian biomes. The Science of Nature, v. 104, p. 1-10, 2017. https://doi.org/10.1007/s00114-017-1456-6

ARRUDA, D.M., SCHAEFER, C.E.G.R., CORREAA, G.R., RODRIGUES, P.M.S., DUQUE-BRASIL, R., FERREIRA-JR, W.G., OLIVEIRA-FILHO, A.T. Landforms and soil attributes determine the vegetation structure in the Brazilian semiarid. Folia Geobotanica, v. 50, p. 175-184, 2015. 
https://doi.org/10.1007/s12224-015-9221-0

BANDA KR, DELGADO-SALINAS A, DEXTER KG, LINARES-PALOMINO R, OLIVEIRA-FILHO A, PRADO D, PULLAN M, QUINTANA C, RIINA R, RODRÍGUEZ GM, WEINTRITT J, ACEVEDORODRÍGUEZ P, ADARVE J, ÁLVAREZ E, ARANGUREN AB, ARTEAGA JC, AYMARD G, CASTAÑO A, CEBALLOS-MAGO N, COGOLLO Á, CUADROS H, DELGADO F, DEVIA W, DUEÑAS H, FAJARDO L, FERNÁNDEZ Á, FERNÁNDEZ MÁ, FRANKLIN J, FREID EH, GALETTI LA, GONTO R, GONZÁLEZ RM, GRAVESON R, HELMER EH, IDÁRRAGA Á, LÓPEZ R, MARCANO-VEGA H, MARTÍNEZ OG, MATURO HM, MCDONALD M, MCLAREN K, MELO O, MIJARES F, MOGNI V, MOLINA D, MORENO NDP, NASSAR JM, NEVES DM, OAKLEY LJ, OATHAM M, OLVERA-LUNA AR, PEZZINI, FF, DOMINGUEZ OJR, RÍOS ME, RIVERA O, RODRÍGUEZ N, ROJAS A, SÄRKINEN T, SÁNCHEZ R, SMITH M, VARGAS C, VILLANUEVA B, PENNINGTON RT. Plant diversity patterns in neotropical dry forests and their conservation implications. Science, v.80, p. 1383-1388, 2016. https://doi.org/10.1126/science.aaf5080

BARROS MF, PINHO, BX, LEÃO T, TABARELLI M. Soil attributes structure plant assemblages across an Atlantic forest mosaic. J. Plant Ecol., v. 11, p. 613-622, 2018. https://doi.org/10.1093/ipe/rtx037

BRINKMAN, R. Ferrolysis, a hydromorphic soil forming process. Geoderma, v. 3, p. 199-206, 1970. https://doi.org/10.1016/0016-7061(70)90019-4

CARTER, M.R. Organic Matter and Sustainability. In: REES, R.M., BALL, B.C., CAMPBELL, C., WATSON, C.A. (Eds.) Sustainable Management of Soil Organic Matter. New York CABI : Publishing, 2000, p. 9-32. https://doi.org/10.1079/9780851994659.0009

DEXTER, K.G., PENNINGTON, R.T., OLIVEIRA-FILHO, A.T., BUENO, M.L., MIRANDA, P.L.S., NEVES, D.M. Inserting Tropical Dry Forests Into the Discussion on Biome Transitions in the Tropics. Frontiers in Ecology Evolution, v. 6, p. 1-7, 2018. https://doi.org/10.3389/fevo.2018.00104

DONAGEMA, G.K., CAMPOS, D.V.B. de, CALDERANO, S.B., TEIXEIRA, W.G., VIANA, J.H.M. Manual de métodos de análise de solos. Rio de Janeiro : Embrapa Solos, 2011.

EMBRAPA. Manual de métodos de análises de solos. Rio de Janeiro : Embrapa. 1997.

EMBRAPA. Banco de dados de Solos. Disponível em:

<https://www.bdsolos.cnptia.embrapa.br/consulta_publica.html>. Acesso em: 18 jun. 2017.

FERREIRA, J.T.P., RIBEIRO FILHO, M.R., RIBEIRO, M.R., SOUZA JÚNIOR, V.S. de, BITTAR, S.M.B., SANTOS, R.G. Planosols Developed in Different Geoenvironmental Conditions in Northeastern Brazil. Revista Brasileira de Ciência do Solo, v. 40, p. 1-18, 2016. https://doi.org/10.1590/18069657rbcs20150131

FONTES, M.P.F., WEED, S.B. Iron Oxides in Selected Brazilian Oxisols: I. Mineralogy. Soil Science Society of American Journal, v. 55, p. 1143-1149, 1991. https://doi.org/10.2136/sssaj1991.03615995005500040040x

GAISER, T.; GRAEF, F.; CORDEIRO, J. C. Water retention characteristics of soils with contrasting clay mineral composition in semi-arid tropical regions. Australian Journal of Soil Research, v. 38, n. 3, p. 523-536, 2000. https://doi.org/10.1071/SR99001

GIONGO, V., CUNHA, T.J.F., MENDES, A.S., GAVA, C.A.T. Carbono no sistem solo-planta no semiárido brasileiro. Revista Brasileira de Geografia Física, v. 6, p. 1233-1253, 2011. https://doi.org/10.26848/rbgf.v4i6.232769

GRAND, S., LAVKULICH, L.M. Effects of Forest Harvest on Soil Carbon and Related Variables in Canadian Spodosols. Soil Science Society of American Journal, v. 76, p. 1816, 2012. https://doi.org/10.2136/sssaj2012.0103

GRÜNEBERG, E., ZICHE, D., WELLBROCK, N. Organic carbon stocks and sequestration rates of forest soils in Germany. Global Change Biology, v. 20, p. 2644-2662, 2014.

https://doi.org/10.1111/gcb.12558

HOORN, C., WESSELINGH, F.P., TER STEEGE, H., BERMUDEZ, M.A., MORA, A., SEVINK, J., SANMARTIN, I., SANCHEZ-MESEGUER, A., ANDERSON, C.L., FIGUEIREDO, J.P., JARAMILLO, C., RIFF, D., NEGRI, F.R., HOOGHIEMSTRA, H., LUNDBERG, J., STADLER, T., SARKINEN, T., ANTONELLI, A., 2010. Amazonia Through Time: Andean Uplift, Climate Change, Landscape Evolution, and Biodiversity. Science, v. 80, p. 927-931, 2010. 
https://doi.org/10.1126/science.1194585

IBGE. Censo Demográfico. 2010.

IUSS WORKING GROUP WRB. World Reference Base for Soil Resources 2014, update 2015. International soil classification system for naming soils and creating legends for soil maps, World Soil Resources Reports. Rome : FAO, 2015.

JONES, M.M., RUOKOLAINEN, K., MARTINEZ, N.C.L., TUOMISTO, H. Differences in topographic and soil habitat specialization between trees and two understorey plant groups in a Costa Rican lowland rain forest. Journal of Tropical Ecology, v. 32, p. 482-497, 2016. https://doi.org/10.1017/S0266467416000419

KAYANO, M.T., ANDREOLI, R. V. Clima da Região Nordeste do Brasil. In: CAVALCANTI, I.F., FERREIRA, N.J., SILVA, M.G.A.J., DIAS, M.A.F.S. (Eds.) Tempo e clima no Brasil. São Paulo : Oficina de textos, 2009, p. 213-234.

MAGNAGO, L.F.S., MARTINS, S. V, SCHAEFER, C.E.G.R., NERI, A. V. Restinga forests of the Brazilian coast: richness and abundance of tree species on different soils. Anais da Academia Brasileira de Ciência, v. 84, p. 807-22, 2012. https://doi.org/10.1590/S0001-37652012000300023

MARQUES, J.J., SCHULZE, D.G., CURI, N., MERTZMAN, S. Trace element geochemistry in Brazilian Cerrado soils. Geoderma, v. 121, p. 31-43. 2004. https://doi.org/10.1016/i.geoderma.2003.10.003

MEDEIROS VB, de OLIVEIRA PE, SANTOS RA, BARRETO AMF, de OLIVEIRA MAT, PINAYA JLD. New holocene pollen records from the Brazilian Caatinga. Anais da Academia Brasileira de Ciência, v.90, p. 2011-2023, 2018. https://doi.org/10.1590/0001-3765201820170161

MENDONÇA, B.A.F., FERNANDES FILHO, E.I., SCHAEFER, C.E.G.R., de MENDONÇA, J.G.F., VASCONCELOS, B.N.F. Soil-vegetation relationships and community structure in a "terra-firme"whitesand vegetation gradient in Viruá National Park, Northern Amazon, Brazil. Anais da Academia Brasileira de Ciência, v. 89, p. 1269-1293, 2017. https://doi.org/10.1590/0001-3765201720160666

MENEZES, R., SAMPAIO, E., GIONGO, V., PÉREZ-MARIN, A. Biogeochemical cycling in terrestrial ecosystems of the Caatinga Biome. Brazilian Journal of Biology, v. 72, p. 643-653, 2012. https://doi.org/10.1590/S1519-69842012000400004

MINICK, K. J.; FISK, M. C.; GROFFMAN, P. M. Soil Ca alters processes contributing to $C$ and N retention in the $\mathrm{Oa} / \mathrm{A}$ horizon of a northern hardwood forest. Biogeochemistry, v. 132, n. 3, p. 343357, 2017. https://doi.org/10.1007/s10533-017-0307-z

MIRANDA, P.L.S. de, OLIVEIRA-FILHO, A.T., PENNINGTON, R.T., NEVES, D.M., BAKER, T.R., DEXTER, K.G. Using tree species inventories to map biomes and assess their climatic overlaps in lowland tropical South America. Global Ecology and Biogeography v, 27, p. 899-912, 2018. https://doi.org/10.1111/geb.12749

MUCINA, L. Biome: evolution of a crucial ecological and biogeographical concept. New Phytologist, v. 222, p. 97-114, 2019. https://doi.org/10.1111/nph.15609

NERI, A. V., SCHAEFER, C.E.G.R., SOUZA, A.L., FERREIRA-JUNIOR, W.G., MEIRA-NETO, J.A.A. Pedology and plant physiognomies in the cerrado, Brazil. Anais da Academia Brasileira de Ciência, v. 85, p. 87-102, 2013. https://doi.org/10.1590/S0001-37652013000100007

NOVOTNY, E. H. et al. Studies of the compositions of humic acids from Amazonian Dark Earth soils. Environmental Science and Technology, v. 41, n. 2, p. 400-405, 2007. https://doi.org/10.1021/es060941x

PELLEGRINI, A. F. A. Nutrient limitation in tropical savannas across multiple scales and mechanisms. Ecology, v. 97, n. 2, p. 313-324, 2016. https://doi.org/10.1890/15-0869.1

PEÑA-CLAROS, M. et al. Soil Effects on Forest Structure and Diversity in a Moist and a Dry Tropical Forest. Biotropica, v. 44, n. 3, p. 276-283, 2012. https://doi.org/10.1111/i.1744-7429.2011.00813.x

PENNINGTON, R.T., LEHMANN, C.E.R., ROWLAND, L.M. Tropical savannas and dry forests. Current Biology, v. 28, p.541-545. 2018. https://doi.org/10.1016/i.cub.2018.03.014

PENNINGTON, R.T., PRADO, D.E., PENDRY, C.A., BOTANIC, R.. Neotropical seasonally dry forests and Quaternary vegetation changes. Journal of Biogeography, v. 27, p. 261-273, 2000. https://doi.org/10.1046/j.1365-2699.2000.00397.x 
PINHEIRO, E.A.R., COSTA, C.A.G., DE ARAÚJO, J.C. Effective root depth of the Caatinga biome. Journal of Arid Environ. v. 89, p. 1-4, 2013. https://doi.org/10.1016/j.jaridenv.2012.10.003

PIRES, C. V, SCHAEFER, C.E.R.G., HASHIGUSHI, A.K., THOMAZINI, A., FILHO, E.I.F., MENDONÇA, E.S. Soil organic carbon and nitrogen pools drive soil C- $\mathrm{CO}_{2}$ emissions from selected soils in Maritime Antarctica. Science of the Total Environment, v. 596, p. 124-135, 2017. https://doi.org/10.1016/j.scitotenv.2017.03.144

POELKING, E.L., SCHAEFER, C.E.R., FERNANDES FILHO, E.I., de ANDRADE, A.M., SPIELMANN, A.A. Soil-landform-plant-community relationships of a periglacial landscape on Potter Peninsula, maritime Antarctica. Solid Earth, v. 6, p. 583-594, 2015. https://doi.org/10.5194/se-6-583-2015

RODRIGUES, P.M.S., SCHAEFER, C.E.G.R., SILVA, J.O., FERREIRA JÚNIOR, W.G., dos SANTOS, R.M., NERI, A.V. The influence of soil on vegetation structure and plant diversity in different tropical savannic and forest habitats. Journal of Plant Ecology, v. 11, p. 226-236, 2016.

https://doi.org/10.1093/jpe/rtw135

SANTOS, H.G.S., JACOMINE, P.K., ANJOS, L.H.C., OLIVEIRA, V.A., LUMBRERAS, J.F., COELHO, M.R., ALMEIDA, J.A., CUNHA, T.J.F., OLIVEIRA, J.B. Sistema brasileiro de classificação de solos. Rio de Janeiro : Embrapa. 2013.

SANTOS, H. G.; CARVALHO JUNIOR, W.; DART, R. O.; AGLIO, M. L. D.; SOUSA, J. S. de; PARES, J. G.; FONTANA, A.; MARTINS, A. L. da S.; OLIVEIRA, A. P. de. Mapa de solos do Brasil.

Disponível em: ftp://geoftp.ibge.gov.br/informacoes_ambientais/pedologia/mapas/brasil/solos.pdf Acesso em: 12 de maio de 2017.

SILVEIRA M. M. L, ARAÚJO, M. S. B., SAMPAIO, E. V. B. Distribuição de fósforo em diferentes ordens de solo do semi-árido da Paraíba e de Pernambuco. Revista Brasileira de Ciencia do Solo, v.30, p. 281-291, 2006. https://doi.org/10.1590/S0100-06832006000200009

SOUZA, J.J.L.L.; ABRAHÃO, W.A.P.; de MELLO, J.W.V.; SILVA, J., COSTA, L.M.; OLIVEIRA, T.S. Geochemistry and spatial variability of metal(loid) concentrations in soils of the state of Minas Gerais, Brazil. Science of the Total Environment, v. 505, p. 338-49, 2015.

https://doi.org/10.1016/j.scitotenv.2014.09.098

SOUZA, J.J.L.L.; FONTES, M. P. F.; GILKES, R.; da COSTA, L.M0;, OLIVEIRA, T.S. Geochemical Signature of Amazon Tropical Rainforest Soils. Revista Brasileira de Ciência do Solo, v. 42, p. 118, 2018b. https://doi.org/10.1590/18069657rbcs20170192

TERRA, M.C.N.S., SANTOS, R.M., PRADO JÚNIOR, J.A., FONTES, M.A., SCOLFORO, J.R.S., FONTES, M.A.L., SCHIAVINI, I., REIS, A.A., BUENO, I.T., Magnago, S., ter Steege, H. Water availability drives gradients of tree diversity, structure and functional traits in the Atlantic-CerradoCaatinga transition, Brazil. Journal of Plant Ecology, v. 11, p. 803-814, 2018. https://doi.org/10.1093/ipe/rty017

TILMAN, D. et al. Diversity and productivity in a long-term grassland experiment. Science, v. 294, n. 5543, p. 843-845, 2001. https://doi.org/10.1126/science.1060391

VALARINI, P. J. et al. Assessment of soil properties by organic matter and EM-microorganism incorporation. Revista Brasileira de Ciência do Solo, v. 27, n. 3, p. 519-525, 2003. https://doi.org/10.1590/S0100-06832003000300013

YEOMANS, J.C., BREMNER, J.M. A rapid and precise method for routine determination of organic carbon in soil. Communications of Soil Science and Plant Analysis, v.19, p. 1467-1476. 1988. https://doi.org/10.1080/00103628809368027

Recebido em: 11/06/2020

Aceito para publicação em: 24/11/2020 